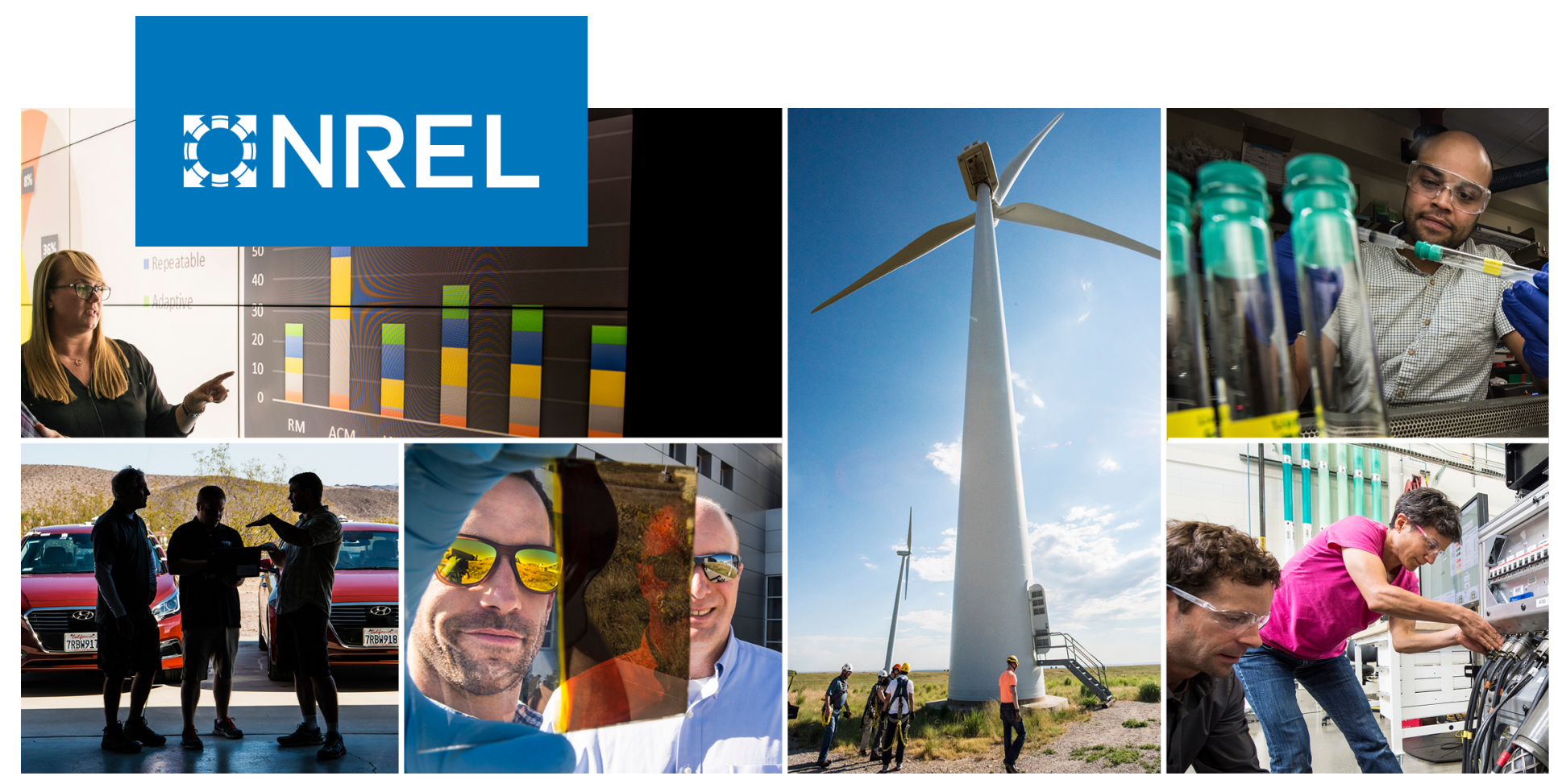

\title{
Demand Response in Bangalore: Implications for Electricity System Operations
}

Madeleine McPherson and Brady Cowiestoll

National Renewable Energy Laboratory

NREL is a national laboratory of the U.S. Department of Energy

Office of Energy Efficiency \& Renewable Energy

Operated by the Alliance for Sustainable Energy, LLC

This report is available at no cost from the National Renewable Energy Laboratory (NREL) at www.nrel.gov/publications.
Technical Report

NREL/TP-6A20-72054

September 2020 


\title{
GNREL
}

\section{Demand Response in Bangalore: Implications for Electricity System Operations}

\author{
Madeleine McPherson and Brady Cowiestoll
}

National Renewable Energy Laboratory

\section{Suggested Citation}

McPherson, Madeleine, and Brady Cowiestoll. 2020. Demand Response in Bangalore: Implications for Electricity System Operations. Golden, CO: National Renewable Energy Laboratory. NREL/TP-6A20-72054. https://www.nrel.gov/docs/fy20osti/72054.pdf.

NREL is a national laboratory of the U.S. Department of Energy Office of Energy Efficiency \& Renewable Energy Operated by the Alliance for Sustainable Energy, LLC

This report is available at no cost from the National Renewable Energy Laboratory (NREL) at www.nrel.gov/publications.

Contract No. DE-AC36-08GO28308
Technical Report

NREL/TP-6A20-72054

September 2020

National Renewable Energy Laboratory 15013 Denver West Parkway Golden, CO 80401 303-275-3000 • www.nrel.gov 


\section{NOTICE}

This work was authored by the National Renewable Energy Laboratory, operated by Alliance for Sustainable Energy, LLC, for the U.S. Department of Energy (DOE) under Contract No. DE-AC36-08GO28308. Funding provided by U.S. Department of Energy Office of Energy Efficiency and Renewable Energy Office of International Affairs (IA). The views expressed in the article do not necessarily represent the views of the DOE or the U.S. Government.

This report is available at no cost from the National Renewable Energy Laboratory (NREL) at www.nrel.gov/publications.

U.S. Department of Energy (DOE) reports produced after 1991 and a growing number of pre-1991 documents are available free via www.OSTI.gov.

Cover Photos by Dennis Schroeder: (clockwise, left to right) NREL 51934, NREL 45897, NREL 42160, NREL 45891, NREL 48097, NREL 46526.

NREL prints on paper that contains recycled content. 


\section{Acknowledgments}

The authors appreciate the data and guidance provided by the Bangalore Electricity Supply Company (BESCOM), especially Anil D'Souza who led engagements with NREL and Jyothi Rani and Lakshmi K, as well as Nikit Abhyankar, Nihan Karali and Aditya Khandekar of the Lawrence Berkeley National Laboratory. The authors also thank David Palchak and Elaine Hale of the National Renewable Energy Laboratory for their thoughtful and helpful reviews, and Elena Thomas-Kerr of the U.S. Department of Energy for her feedback and support throughout the project. This research was funded by the U.S. Department of Energy under proposal number IAGTG India DR- FY16-01. We would also like to acknowledge USAID for their support and funding of the Greening The Grid modeling efforts (http://www.nrel.gov/india-grid-integration) which were heavily used in this work. Monali Hazra (USAID) also provided critical support throughout the project.

This work was authored by Alliance for Sustainable Energy, LLC, the manager and operator of the National Renewable Energy Laboratory for the U.S. Department of Energy (DOE) under the Field Work Proposal No. IA-GTG India SMART Cities DR-FY16-01 titled, GTG India Demand Response Analysis. Funding was provided by the United States Department of Energy Office of International Affairs. The views expressed in the article do not necessarily represent the views of the DOE or the U.S. Government. The U.S. Government retains and the publisher, by accepting the article for publication, acknowledges that the U.S. Government retains a nonexclusive, paidup, irrevocable, worldwide license to publish or reproduce the published form of this work, or allow others to do so, for U.S. Government purposes. 


$\begin{array}{ll}\text { List of Acronyms and Abbreviations } \\ \text { BESCOM } & \text { Bangalore Electricity Supply Company } \\ \mathrm{CO}_{2} & \text { carbon dioxide } \\ \text { CC } & \text { combined cycle } \\ \text { CEA } & \text { Central Electricity Authority (of India) } \\ \text { CHT } & \text { commercial high-tension } \\ \text { CLT } & \text { commercial low-tension } \\ \text { CT } & \text { combustion turbine } \\ \text { CTU } & \text { Central Transmission Utility (of India) } \\ \text { DR } & \text { demand response } \\ \text { GWh } & \text { gigawatt-hours } \\ \text { HT } & \text { high-tension line (bulk power purchasers with 11 kV or more) } \\ \text { IHT } & \text { industrial high-tension } \\ \text { ILT } & \text { industrial low-tension } \\ \text { INR } & \text { Indian rupees } \\ \text { KV } & \text { kilovolt } \\ \text { KPTCL } & \text { Karnataka Power Transmission Corporation Limited } \\ \text { LBNL } & \text { Lawrence Berkeley National Laboratory } \\ \text { LT } & \text { low-tension line (400-V to 230-V connections) } \\ \text { nDR } & \text { no DR (scenario) } \\ \text { NREL } & \text { National Renewable Energy Laboratory } \\ \text { PV } & \text { photovoltaic } \\ \text { USD } & \text { U.S. dollars } \\ \text { V } & \text { volt } \\ \text { VRE } & \text { variable renewable energy } \\ \text { wDR } & \text { with DR (scenario) } \\ & \end{array}$




\section{Abstract}

Recent Greening the Grid studies for India highlight the benefits of flexible resources for integrating variable renewable energy onto India's electricity system. India's ambitious renewable energy targets, which are particularly focused on renewable resource states such as Karnataka, will face fewer challenges when combined with new planning and operational strategies and technologies. This report explores one such strategy_demand response-by which the system operator shifts load throughout a day to minimize system wide production costs. To explore this strategy, we added demand response resources to Karnataka's electricity system in a production cost model of India, using load shifting potential analyzed by Lawrence Berkeley National Labs. We then investigated the impacts of increasing demand response capacity under several renewable resource scenarios. Our results show the addition of demand response enables fuel shifting from high-marginal-cost and emissions-intensive subcritical coal and diesel generation to zero-marginal-cost and emissions-free renewable generation.

Accordingly, the value that demand response provides to the system increases as the renewable penetration increases. In addition to reducing production costs and emissions, demand response reduces the time that thermal generators spend at their minimum output levels, which typically represents a less efficient and costlier operational state. Agricultural load shifting provides greater value to the system than residential, commercial, or industrial loads. Agricultural demand response is more flexible than other sectors because it is not exposed to subdaily operational constraints and it can operate for more hours per day without impacting customer satisfaction. Further, the first increment of demand response that is added to a system provides the greatest value; further additions provide additional benefits but have a decreasing impact. The insights we discuss could be leveraged by system planners and operators in other jurisdictions, particularly those facing significant renewable energy penetrations, to develop their own demand response programs. 


\section{Table of Contents}

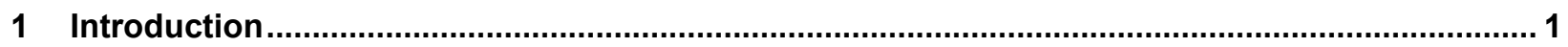

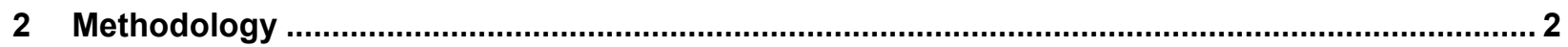

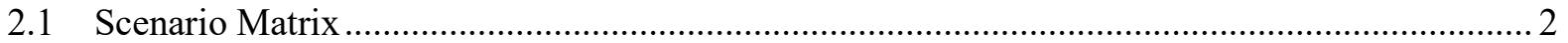

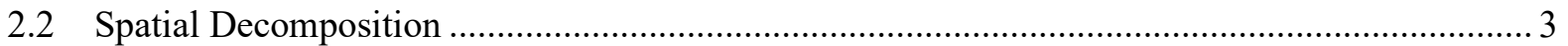

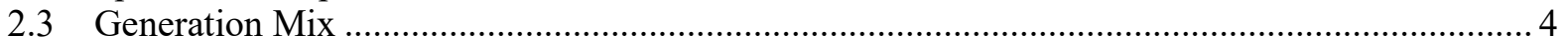

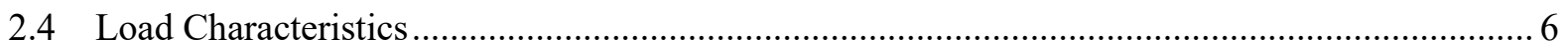

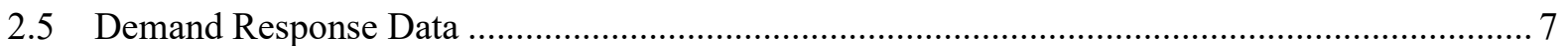

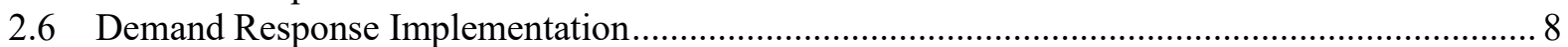

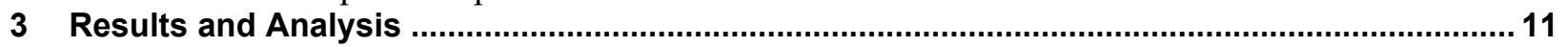

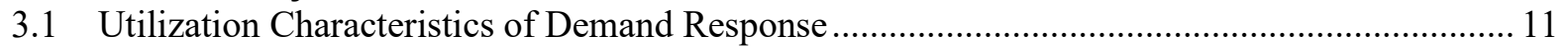

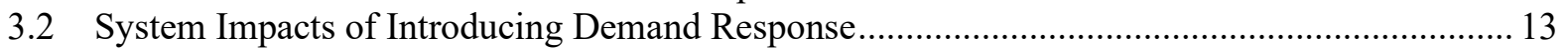

3.3 System Operational Implications of Integrating Demand Response ........................................ 16

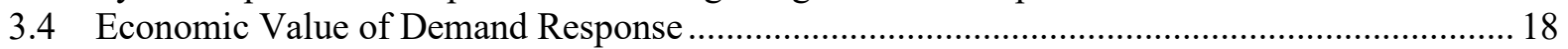

3.5 Impact of Demand Response in Different Renewable Energy Scenarios ................................. 20

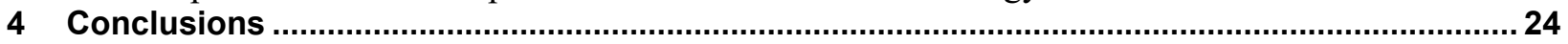

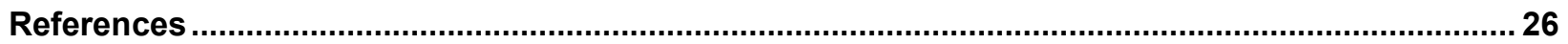




\section{List of Figures}

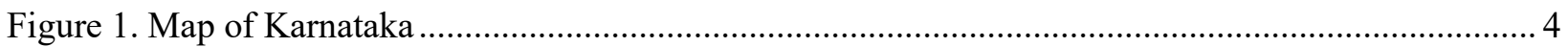

Figure 2. Installed capacity across the RE scenarios (without DR) ..................................................... 4

Figure 3. Installed capacity across the DR scenarios (High Solar case) .................................................. 5

Figure 4. Merit order curve of Karnataka's thermal generation fleet (excluding zero-marginal-cost

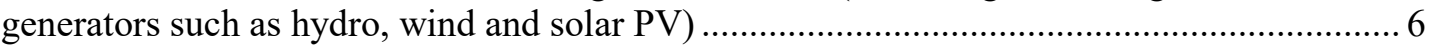

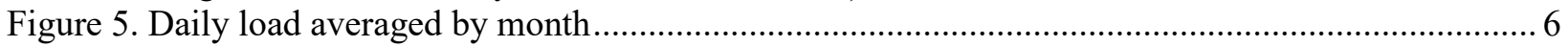

Figure 6. Demand response availability in the Low DR, Medium DR, and High DR scenarios................ 7

Figure 7. Demand response constraints: (a) power availability, (b) recovery time, (c) recovery availability,

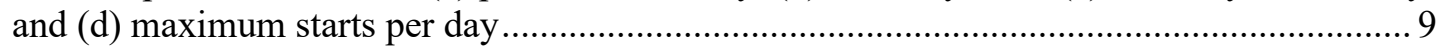

Figure 8. Demand response activity per end use in the Low DR, Medium DR, and High DR scenarios... 11

Figure 9. Daily DR operations averaged for each hour of the day over all nodes and all end uses in the High DR and Low DR, HighSR scenarios, as compared to net load ................................... 12

Figure 11. Monthly DR utilization per end use for all end uses (top) and excluding agriculture (bottom) 13

Figure 12. Karnataka's baseline generation profile (left) compared to the change in generation due to the introduction of DR (right) in the High Solar scenario .......................................................... 14

Figure 13. Marginal reduction in production costs per unit of DR activity for increasing DR availability 15

Figure 14. Marginal reduction in $\mathrm{CO}_{2}$ emissions due to the introduction of DR, per unit of DR activity.. 16

Figure 15. Impact of DR on diesel operations: Plant load factor (left), number of starts (center), and percentage of time at minimum stable level (right) ............................................................... 16

Figure 16. Impact of DR on supercritical coal (top) and subcritical coal (bottom) operations for plant load factor (left), number of starts (center), and percentage of time at minimum stable level (right)

Figure 17. Capacity credit of each DR end use...………………………………………………….... 18

Figure 17. Demand response revenues per MWh of DR generation, by end use for each DR scenario..... 20

Figure 20. Generation in each RE scenario in Karnataka, before DR is added to the system ................... 21

Figure 21. Change in generation after introducing the medium DR availability scenario for each RE

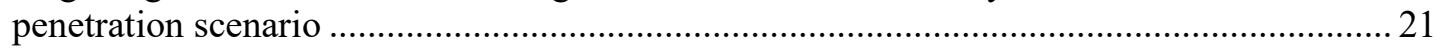

Figure 22. Annual activity of each DR end use in the Medium DR scenario, for each RE

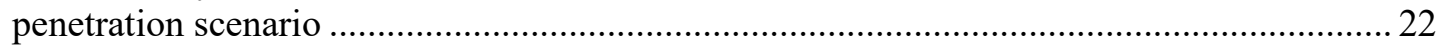

Figure 23. Daily DR operations averaged overall all nodes and end uses as compared to the net load in the Medium DR scenario, for each RE penetration scenario ................................................... 23

\section{List of Tables}

Table 1. India-Wide Renewable Energy Penetration Scenarios 3

Table 2. Assumptions of Demand Response End-Use Constraints 


\section{Introduction}

India has established a goal of having 175 gigawatts $(\mathrm{GW})$ of renewable generation, including 60 $\mathrm{GW}$ of wind and $100 \mathrm{GW}$ of solar, onto the electricity system by 2022 . The integration of large amounts of renewable energy (RE) onto the grid can transform the way that the grid operates, and inflexible systems in particular may have more challenges due to the inherent uncertainty and variability of variable renewable energy (VRE) resources. As a result, technologies and strategies that provide flexibility for power systems are becoming increasingly pertinent in jurisdictions with large VRE penetrations. Demand response (DR) could represent an important part of the flexibility toolkit, particularly over short durations. However, tools to analyze the potential of such technologies in the context of high RE futures are not well established and miss key aspects of real-world operation of DR. As a result, we refined methodologies for this analysis that can capture the value of DR in real-world systems at operational timescales, and we applied these to India as a case study.

This report builds on the recently published Greening the Grid $^{1}$ series, which assesses the impacts of increasing VRE penetrations on India's electricity system at the national level in Volume One (Palchak, Cochran, Ehlen, McBennett, Milligan, Chernyakhovskiy, Deshmukh, et al. 2017a) and at the regional level in Volume Two (Palchak, Cochran, Ehlen, McBennett, Milligan, Chernyakhovskiy, Deshmukh, et al. 2017b). Volume One focuses on renewable energy integration impacts and policy at the national scale by simplifying intrastate transmission infrastructure. Volume Two (Palchak, Cochran, Ehlen, McBennett, Milligan, Chernyakhovskiy, Deshmukh, et al. 2017b) focuses on the regional scale, and six high-VRE states - in particular, Andhra Pradesh, Gujarat, Karnataka, Maharashtra, Rajasthan, and Tamil Nadu — by including intrastate transmission. While the Greening the Grid series addresses many aspects of VRE integration, the strategies that increase India's grid flexibility are most applicable to this report. Palchak et al. (Palchak, Cochran, Ehlen, McBennett, Milligan, Chernyakhovskiy, Deshmukh, et al. 2017b) find that reducing the minimum coal generation level from $70 \%$ to $55 \%$ to $40 \%$ of rated capacity reduces curtailment in India's Southern Region from $8.4 \%$ to $4.9 \%$ to $3.0 \%$, respectively. Additionally, they find that regionally coordinating dispatch makes more efficient use of least-cost generation which in turn reduces production costs (Palchak, Cochran, Ehlen, McBennett, Milligan, Chernyakhovskiy, Deshmukh, et al. 2017b). Our DR analysis builds on these findings by exploring the introduction of flexibility on the demand side of the electricity system as an additional mechanism to facilitate VRE integration.

Demand response participants can change their electricity patterns in three ways: temporally shift energy consumption, reduce energy consumption, or self-generate (Siano 2014). Demand response in this report refers to the first: the ability of different load types, referred to as "end uses," to change their load according to a signal from either a grid operator or an aggregator of many load participants. Many different types of load, such as lighting, heating, refrigeration or industrial processes, can participate in DR programs by reducing or shifting their load. And, DR

\footnotetext{
${ }^{1}$ Greening the Grid is a U.S.-led project that offers information and guidance to help developing countries define and implement grid-integration roadmaps. For more information, see greeningthegrid.org.
} 
can have numerous system benefits, including increased power system flexibility, increased economic efficiency, and reduced generation capacity requirements (O'Connell et al. 2014).

To facilitate an analysis of DR, which is a geographically diffuse resource, this analysis focused on Bangalore and its surrounding area in the southern state of Karnataka. Feeder-specific load profiles, broken out by sector and end use, have been developed by Lawrence Berkeley National Laboratory (LBNL) for the Bangalore Electricity Supply Company (BESCOM) territory with 15-minute temporal resolution (Karali, Abhyankar, and Khandekar 2019). These load profiles were used to determine the maximum DR available at each time step from each end use. Additionally, constraints specific to each end use and sector limited the responsiveness of load according to consumer tolerance assumptions. Finally, the impacts of this potential demand responsiveness, for each sector-specific end use, were evaluated with high spatial and temporal detail.

The remainder of this report is organized as follows. Section 2 introduces the methodology employed in the analysis, including the scenario matrix, the spatial decomposition process, Karnataka's generation fleet and load characteristics, and the DR data and representation. Assumptions and methods that overlap with the Greening the Grid series, such as generatorspecific constraints or transmission topology, are not reproduced in Section 2 to maintain brevity and reduce repetition. Section 3 describes the results of integrating DR in BESCOM territory, focusing on the system-wide impacts, the operational characteristics, and value of DR. Finally, Section 4 concludes the report.

\section{Methodology}

This analysis employs the commercial production cost model (PLEXOS) and the database of India's generation and transmission infrastructure that was developed as part of the Greening the Grid study. Accordingly, the results from this analysis can be given broader context with the Greening the Grid results. Details about the assumptions and constraints included in the India electricity system model can be found in the Greening the Grid reports.

PLEXOS is a software package developed by Energy Exemplar that simulates unit commitment and economic dispatch decisions using mixed-integer programming (Energy Exemplar 2018). Generators are committed and dispatched to achieve the least-cost generation schedule, while adhering to transmission, generation, and resource constraints. The following section elaborates on additional steps and adjustments that were made in the current analysis to facilitate the focus on DR modeling and impacts.

\subsection{Scenario Matrix}

The primary research question posed in this analysis is, how can introducing DR facilitate VRE integration? To address this question, two sets of scenarios are used. First, five renewable energy penetration scenarios, which are consistent with the Greening the Grid scenarios, explore increasing VRE penetrations in India, with penetrations shown in Table 1. 
Table 1. India-Wide Renewable Energy Penetration Scenarios

\begin{tabular}{|ll|rrr|rrr|}
\hline Name & Abbreviation & \multicolumn{3}{|c|}{ India } & \multicolumn{3}{|c|}{ Karnataka } \\
Solar & $\begin{array}{c}\text { Wind } \\
\text { (GW) }\end{array}$ & $\begin{array}{c}\text { Total VRE } \\
\text { Penetration }\end{array}$ & $\begin{array}{c}\text { Solar } \\
\text { (GW) }\end{array}$ & $\begin{array}{l}\text { Wind } \\
\text { (GW) }\end{array}$ & $\begin{array}{c}\text { Total VRE } \\
\text { Penetration }\end{array}$ \\
\hline High RE & HighRE & 150 & 100 & $33 \%$ & 19 & 10 & $60 \%$ \\
$\begin{array}{l}\text { High } \\
\text { Wind }\end{array}$ & HighWD & 60 & 100 & $26 \%$ & 6 & 10 & $53 \%$ \\
$\begin{array}{l}\text { High } \\
\text { Solar }\end{array}$ & HighSR & 100 & 60 & $22 \%$ & 11 & 6 & $48 \%$ \\
$\begin{array}{l}\text { Medium } \\
\text { RE }\end{array}$ & MedmRE & 20 & 50 & $12 \%$ & 2 & 5 & $28 \%$ \\
$\begin{array}{l}\text { No New } \\
\text { RE }\end{array}$ & NNewRE & 0 & $28 \mathrm{GW}$ & $4.8 \%$ & 0 & 3 & $13 \%$ \\
\hline
\end{tabular}

Second, three DR scenarios in addition to the baseline No DR scenario are developed for BESCOM's territory in Karnataka, based on the responsiveness of an end use. Here responsiveness refers to the ability to control the power draw of an electrical load, such as air conditioning (AC) or commercial heating. The "availability" is the amount of the resource that can be controlled at any point in time. Sections 2.5 and 2.6 explain the DR methods. The DR scenarios represent a linear increase in participation of responsive loads: High DR assumes $100 \%$ participation of responsive end uses, Medium DR assumes 67\% participation of responsive end uses, and Low DR assumes 33\% participation of responsive end uses.

\subsection{Spatial Decomposition}

Karnataka, located in the Southern region of India (Figure 1), is electrically connected with each of its neighboring states. Therefore, interstate imports and exports between Karnataka and its neighbors must be accounted for when assessing the statewide generation mix. However, the highly spatially resolved nature of DR requires a nodal model representation. To capture both interstate transmission flows and nodal resolution, this analysis employs a two-stage modeling approach. In the first stage, all of India's generators and load centers are modeled assuming state-wide transmission aggregation outside Karnataka and excluding DR detail. ${ }^{2}$ The stage one model is simulated for an entire year. Next, a state-level model of Karnataka is dispatched, again for a full year, which introduces DR potential into the BESCOM territory in the area around Bangalore. The interstate transmission flows, statewide contribution to reserves, and centrally coordinated hydro dispatched were fixed within this second stage according to the stage one results. This spatial decomposition of the problem enables us to capture both the national impacts of a connected region and the local impacts of a dispersed resource such as DR. The outputs of

\footnotetext{
2 The modeled demand response resource is small enough that it will not impact imports and exports from Karnataka. If larger demand resources were considered, this assumption might be invalid.
} 
the stage two model, including those that are held over from stage one, comprise the discussion in the results section (Section 3).

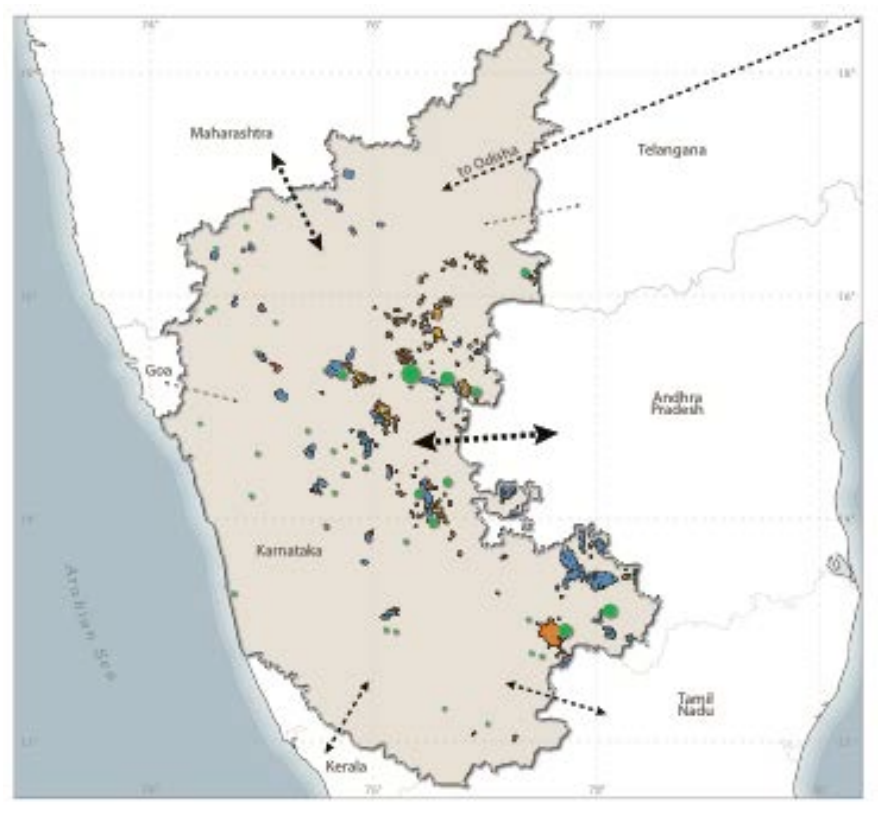

Figure 1. Map of Karnataka

The maps show Karnataka in India's Southern Region and its electrical exchanges with other regions Source: (Palchak, Cochran, Ehlen, McBennett, Milligan, Chernyakhovskiy, llya, et al. 2017)

\subsection{Generation Mix}

Karnataka's existing generation fleet consists primarily of coal, hydro, and wind resources, as well as some nuclear and diesel. The five RE penetration scenarios add progressively greater wind and solar capacity, while the existing fleet is held constant. Figure 2 shows each RE scenario explored in this analysis, before DR is added to the system. In Karnataka, the "Other" category shown in figures consists primarily of diesel generation.

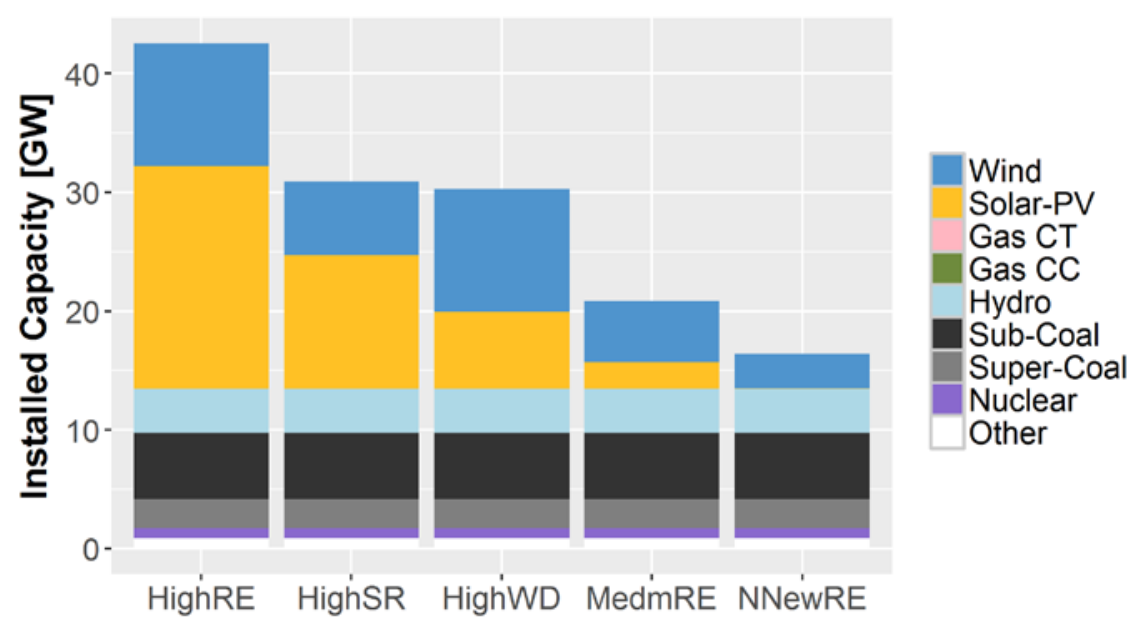

Figure 2. Installed capacity across the RE scenarios (without DR)

$$
\mathrm{CT}=\text { combustion turbine }, \mathrm{CC}=\text { combined cycle }
$$


The DR capacity added to Karnataka's system in each of the DR scenarios-Low DR, Medium DR, and High DR - is shown in Figure 3 for the High Solar renewable energy scenario; the High Solar case is India's current policy and is thus the focus of this report. The DR capacity is small relative to Karnataka's installed capacity for several reasons. First, BESCOM's territory includes Bangalore and the surrounding area but excludes the remainder of Karnataka. Second, numerous demand end uses are not considered in this study, and thus, DR includes only a fraction of the full system's load (2.8\% in the High DR scenario). And third, significant RE capacity has been added to the system without removing conventional resources, thereby increasing the overall system capacity. Practically, redundant thermal generators would likely retire.

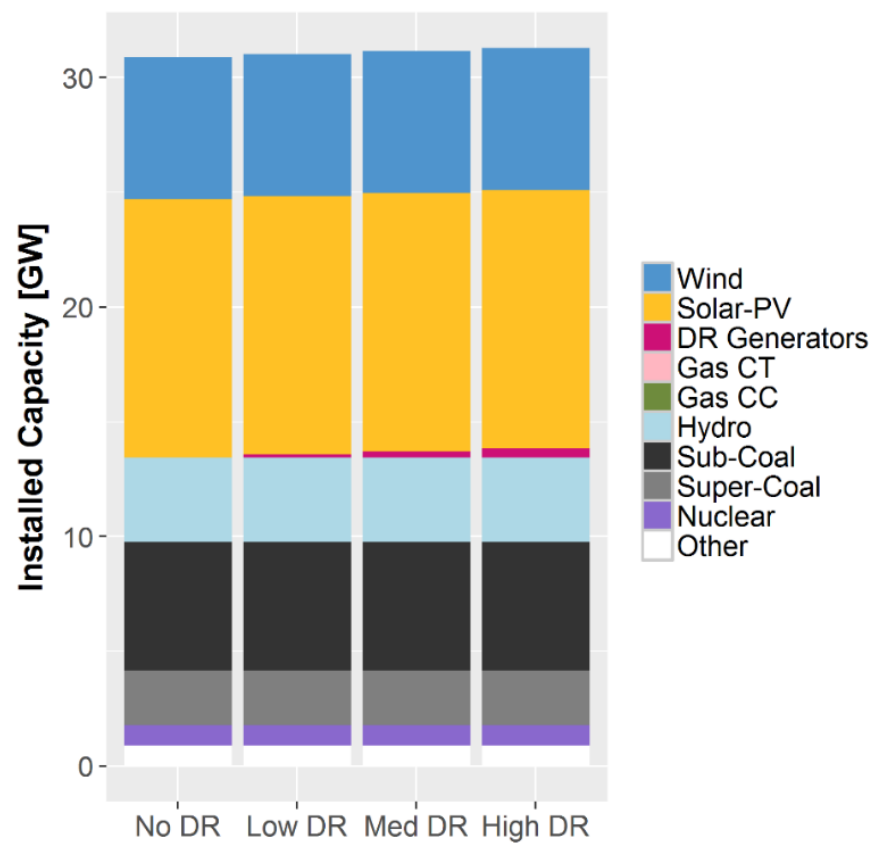

Figure 3. Installed capacity across the DR scenarios (High Solar case)

"Other" assets followed by subcritical coal assets have the highest marginal costs of Karnataka's generation types. On the other hand, supercritical coal is the least-cost non-renewable generation type in Karnataka, as shown in Figure 4. Zero-marginal-cost generators including hydro, wind, and solar photovoltaics (PV) have been excluded from Figure 4 for clarity. While dispatch does not always occur exactly along the merit order curve, this curve indicates which generator types have lower operational costs and are therefore desirable from a least-cost optimization perspective (assuming all other constraints are non-binding). This becomes important for understanding the impacts of DR, as DR resources enable fuel shifting to lower cost resources. At high VRE penetrations, this tends to enable renewable integration, but it also can include reduction of high cost thermal generators for lower cost thermal generators. 


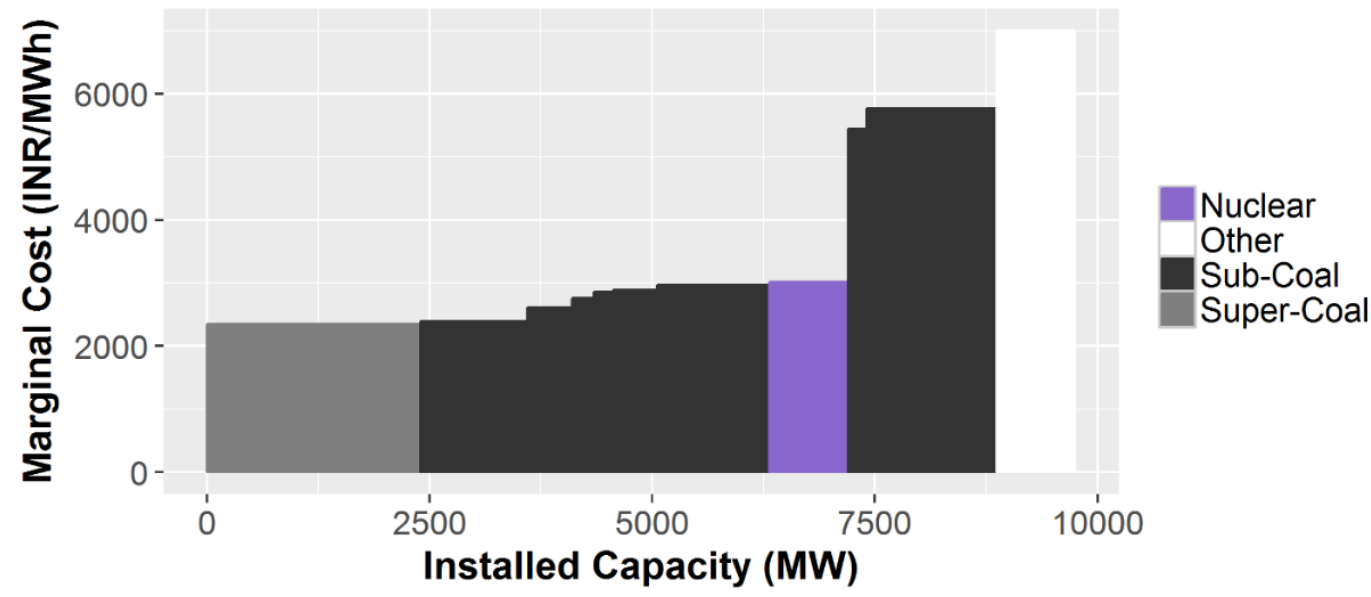

Figure 4. Merit order curve of Karnataka's thermal generation fleet (excluding zero-marginal-cost generators such as hydro, wind and solar PV)

\subsection{Load Characteristics}

Karnataka's baseline load, before DR, peaks in the morning between 8 a.m. and 9 a.m. and again in the evening from 6 p.m. to 7 p.m. (Palchak, Cochran, Ehlen, McBennett, Milligan, Chernyakhovskiy, Deshmukh, et al. 2017b)(Palchak, Cochran, Ehlen, McBennett, Milligan, Chernyakhovskiy, Deshmukh, et al. 2017a). These daily load patterns are consistent throughout the year, as shown in Figure 5. However, the magnitude of the load curve shifts seasonally, peaking between January and April and falling between August and October. These load profiles, broken out by node and disaggregated into end uses as described in (Karali et. al. Forthcoming), are used to inform the quantity and type of electricity consumption that is potentially available as a DR resource during any given time in the day or season of the year.

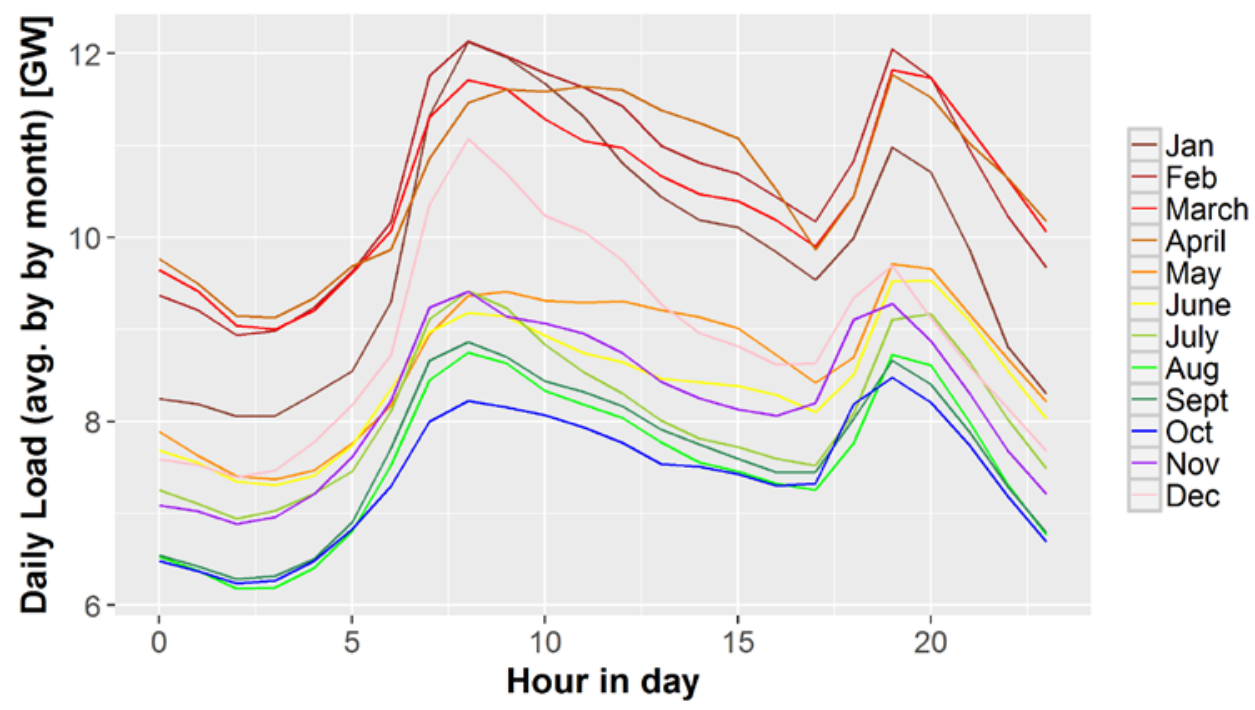

Figure 5. Daily load averaged by month. Note that $y$-axis does not go to zero. 


\subsection{Demand Response Data}

Lawrence Berkeley National Laboratory (LBNL) developed DR timeseries data by combining 15-minute feeder-level generation data with survey data detailing feeder-specific end-use capacity. A detailed description and of LBNL's methodology and results can be found in (Karali et. al. Forthcoming). The LBNL data set divides load data by sector, including residential, commercial, industrial, and agriculture, as well as by end uses within each sector. Only a subset of specific end uses are appropriate for participating in a DR program, including residential AC and refrigerators, commercial kitchen appliances and space cooling, industrial motors and AC, and agricultural loads. These end uses are characterized by the ability to shift load for a short period of time without impacting customers. Other end uses are either not able to be shifted or would have a negative impact on customers. Such end uses included in the end-use survey but assumed to be excluded from the responsive load profiles include lighting, entertainment, electric water heaters, ${ }^{3}$ and ceiling fans. The annual load participating in DR across the BESCOM territory for each of these end uses is shown for each DR scenario in Figure 6. Demand response availability refers to the fraction of load that is assumed to participate in the DR program, and thus is eligible to be shifted. Note that commercial and industrial sectors have been divided into (1) low-tension (LT) connections (400-V to $230-\mathrm{V}$ lines), represented by commercial lowtension (CLT) and industrial low-tension (ILT) respectively in Figure 6, and (2) high-tension (HT) connections (11 kV line or above), represented by CHT and IHT respectively in the figure. Agricultural and HT industrial loads dominate Karnataka's responsive load, while LT commercial and industrial loads are minimal.

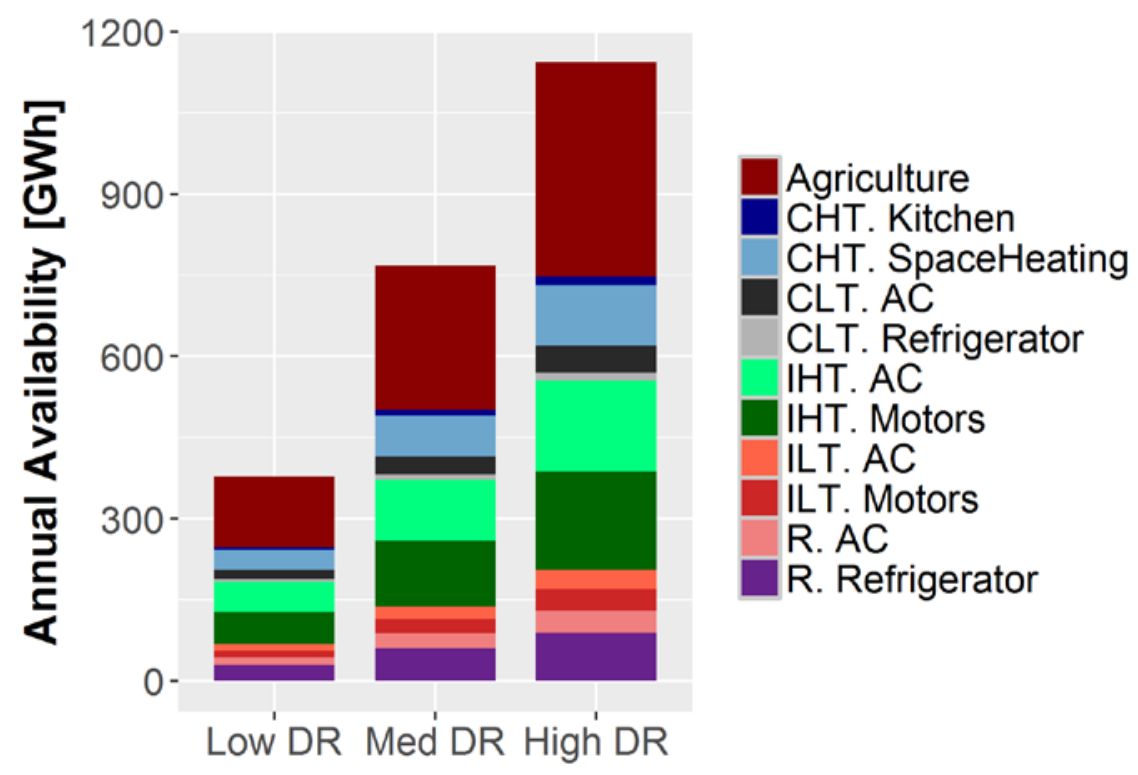

Figure 6. Demand response availability in the Low DR, Medium DR, and High DR scenarios In the High DR scenario, all of the demand responsive load participates in the DR program.

\footnotetext{
${ }^{3}$ In India, small-tank, electric water heaters are the most common type of water heater. We assumed these are unavailable for demand response because there is limited storage to enable energy shifts.
} 


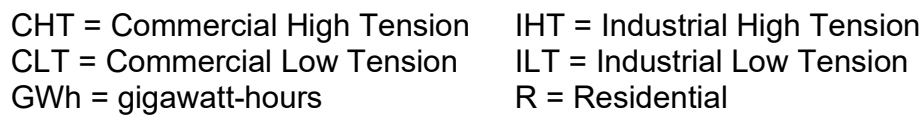

\subsection{Demand Response Implementation}

Demand response, which is a subset of a broader demand side management category, can shift load from one part of the day to a different part of the day, but it must be energy-neutral over a short period to maintain customer comfort levels or needs. Demand response scheduling is constrained by consumers' degree of tolerance for having their load shifted.

Four constraints have been developed and implemented in PLEXOS to represent consumeracceptable load shifting and are applied to "DR events," which are instances in which the system operator calls on the DR program to shift load. The event includes both the call to an end use to reduce load as well as subsequent increases in load, termed recovery, to regain the energy lost. This represents the shift of energy, such as by delaying an industrial process or increasing AC needs to return temperatures to a desired set point. The recovery time is the time from beginning of a DR event to energy neutrality. First, the load available for shifting is limited by appliance operational schedules; in other words, a DR program can only reduce power consumption to the extent that an end use is consuming power at each moment in time (Figure 7a). Second, the maximum allowable duration of any single DR event and the amount of time between a DR event and its subsequent energy recovery, termed the recovery time, is limited; consumers limit the time they are willing to wait before a reduction in load is recovered (Figure $7 \mathrm{~b}$ ). Third, the subsequent energy recovery after a DR-induced reduction in consumption is limited by the installed equipment capacity for that particular end use (Figure 7c). Finally, the number of DR events that can occur per day, implemented as the maximum starts per day, is constrained based on consumer preferences (Figure 7c). Taken together, these constraints significantly impact the operation of DR from a system operator's perspective. Table 2 shows the end-use specific assumptions pertaining to each of these constraints, including the recovery times, the maximum allowable DR event duration (termed its maximum use time), and the maximum allowable DR events per day. 


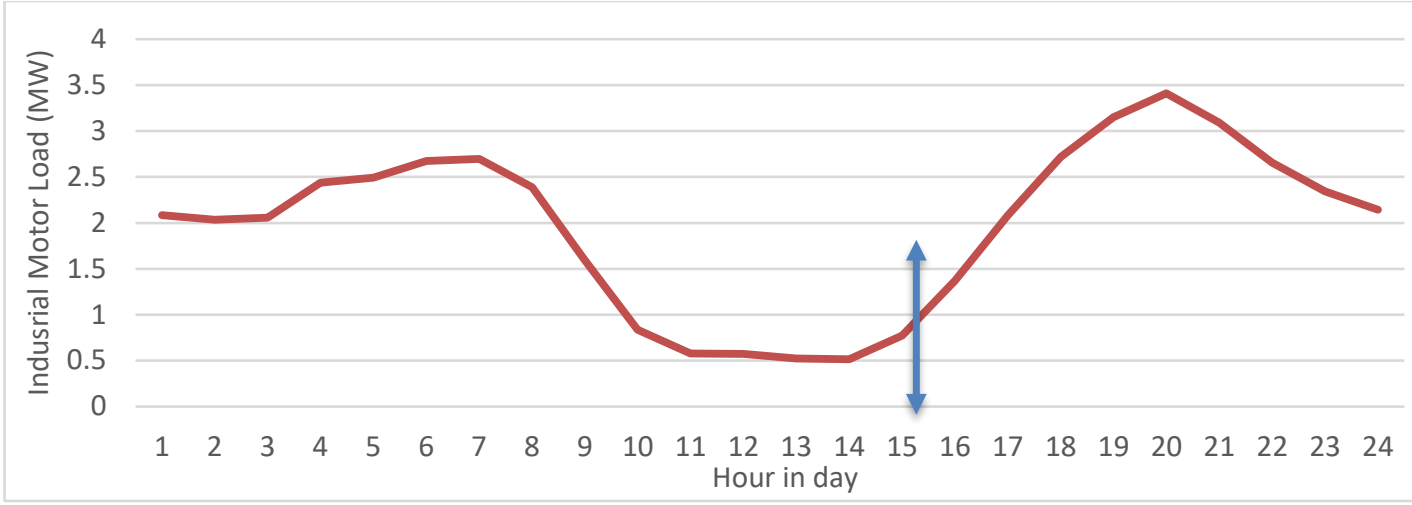

(a)

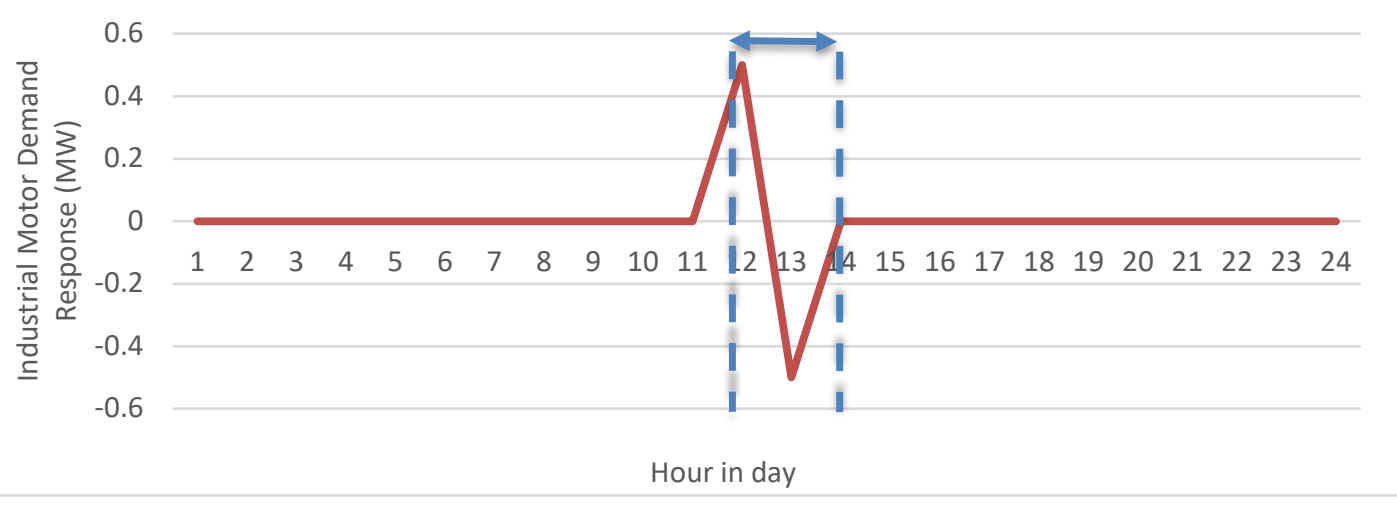

(b)

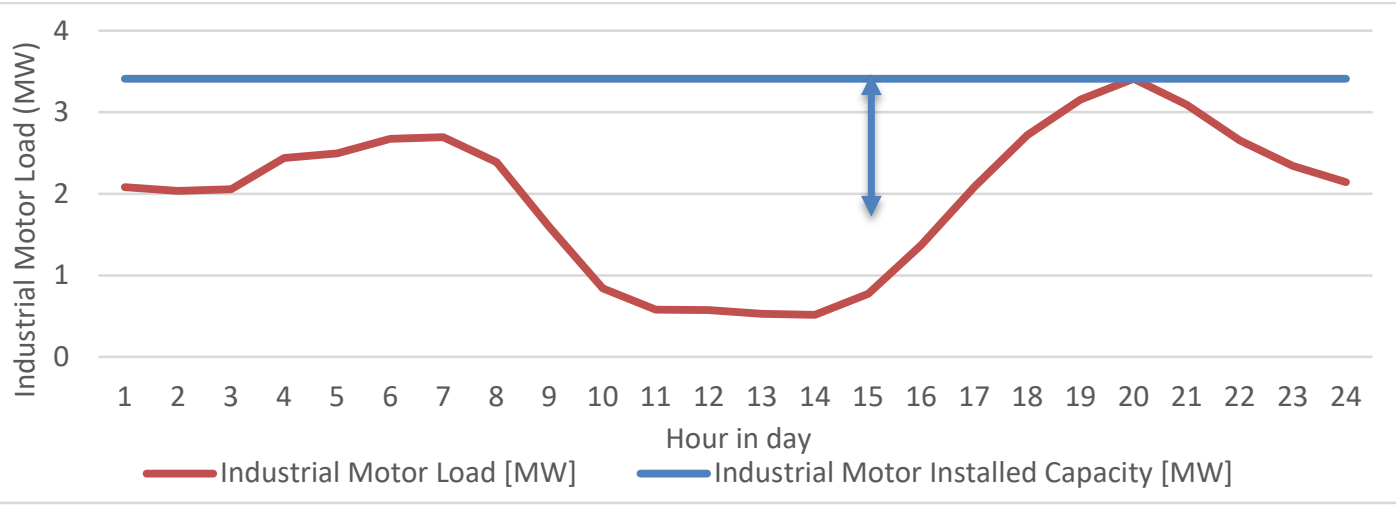

(c)

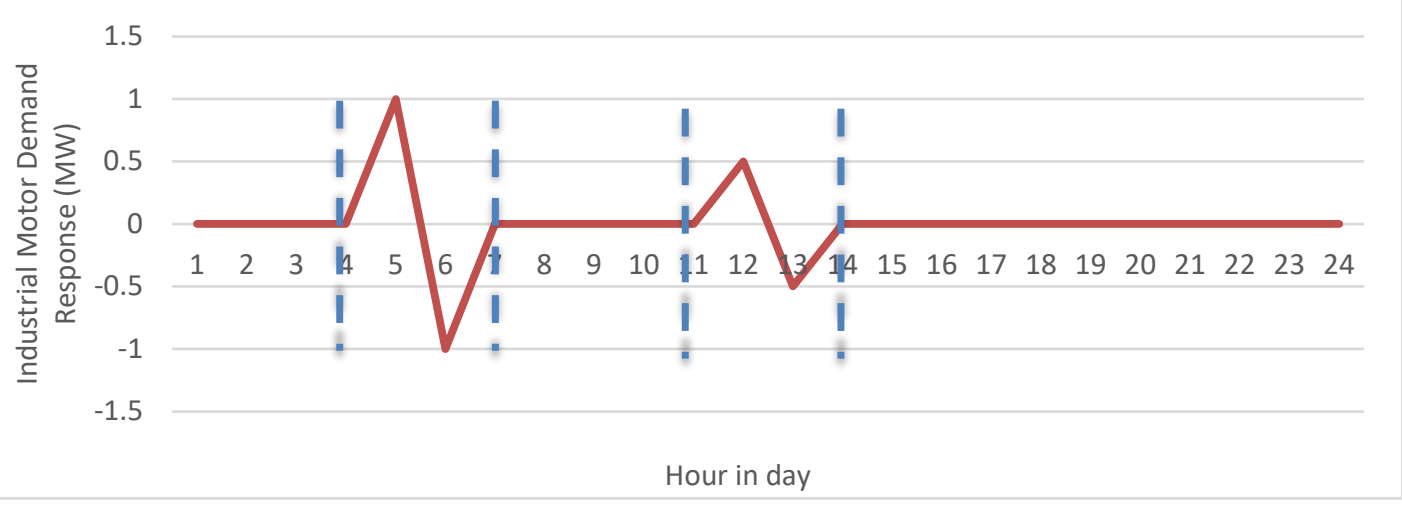

Figure 7. Demand response constraints: (a) power availability, (b) recovery time, (c) recovery availability, and (d) maximum starts per day 
Agriculture is modeled differently from the other end uses to reflect the specific nature of its operation in Karnataka's electricity system. More specifically, agricultural load shifting will tolerate DR events and recovery that last up to 24 hours, which represents a much more relaxed recovery time constraint than that of the other end uses. Additionally, each DR event must last between three and seven hours, which yields more overall energy availability per unit of installed capacity than other end uses. In sum, agriculture has higher power availability (Figure 6), more energy availability (per the longer maximum use per DR event), and more-relaxed recovery constraints (per the longer recovery time) than other end uses.

Table 2. Assumptions of Demand Response End-Use Constraints

\begin{tabular}{|c|c|c|c|c|c|}
\hline Sector & End Use & $\begin{array}{c}\text { Recovery } \\
\text { Time } \\
\text { (hours) }\end{array}$ & $\begin{array}{c}\text { Minimum } \\
\text { Uptime } \\
\text { (hours) }\end{array}$ & $\begin{array}{l}\text { Maximum } \\
\text { Use Time } \\
\text { (time/use) }\end{array}$ & $\begin{array}{c}\text { Maximum } \\
\text { Events } \\
\text { (per day) }\end{array}$ \\
\hline \multirow{2}{*}{ Residential } & $A C$ & 4 & - & 15 minutes & 2 \\
\hline & Refrigerator & 4 & - & 15 minutes & 2 \\
\hline \multirow{2}{*}{$\begin{array}{l}\text { Commercial } \\
\text { (LT and HT) }\end{array}$} & Kitchen appliances & 4 & - & 1 hour & 2 \\
\hline & Space cooling & 4 & - & 15 minutes & 2 \\
\hline \multirow{2}{*}{$\begin{array}{l}\text { Industrial } \\
\text { (LT and HT) }\end{array}$} & Motors & 2 & - & 15 minutes & 2 \\
\hline & Air conditioning & 4 & - & 15 minutes & 2 \\
\hline Agriculture & Agriculture & 24 & 3 & 7 hours & 1 \\
\hline
\end{tabular}

The roundtrip efficiency for DR is assumed to be $100 \%$; that is, shifting load is assumed to not incur additional energy requirements. This issue is complicated, with DR events potentially requiring more or less energy than was reduced in the DR event (O'Connell et al. 2015). For the sake of simplicity, we assume all events are energy neutral. 


\section{Results and Analysis}

Demand response is dispatched to minimize the system-wide production cost while adhering to relevant constraints, as described in Table 2. This section explores the dispatch of DR and the impacts of the operational limitations of each end use. System-wide impacts on operations and key performance metrics are also described. Finally, the value DR brings to the system is compared to the revenues that a DR aggregator could expect to receive. Section 3.1 focuses on the High Solar case, which represents India's intended capacity expansion plan for 2022. The system-wide impacts of different renewable resource scenarios are discussed in Section 3.5.

\subsection{Utilization Characteristics of Demand Response}

Agricultural DR is utilized significantly more than other DR end uses, providing $83 \%-84 \%$ of all DR generation in BESCOM territory, as shown in Figure 8; the utilization rates of other sectors - commercial, industrial, and residential - are lower. Demand response utilization rates increase proportionally as their availability increases in the Low DR, Medium DR, and High DR scenarios. This indicates that the need for flexibility to help integrate variable generation is not saturated by the total available DR in Bangalore.

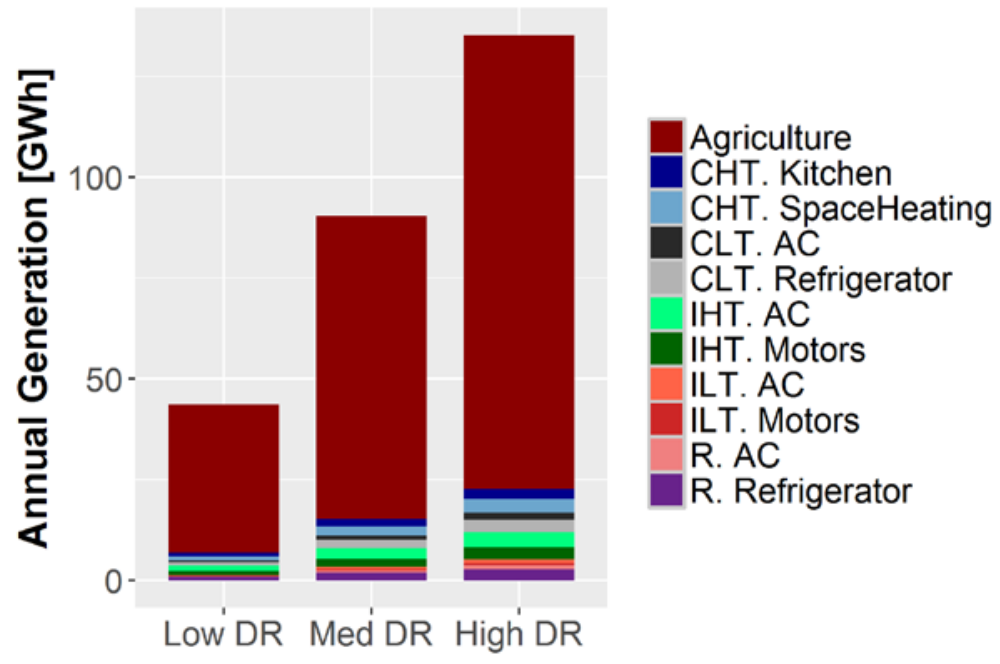

Figure 8. Demand response activity per end use in the Low DR, Medium DR, and High DR scenarios

The significantly higher utilization rates observed for agriculture, as compared to the other sectors, is largely due to its more relaxed operational constraints. As discussed above, an agricultural DR event can persist for longer than other end uses; an agricultural DR event can last 3-7 hours per day instead of just $0.5-2$ hours of daily DR activity for the other end uses. Such longer daily operational periods effectively increase the overall energy available from agricultural DR. Additionally, agriculture is the largest source of load available for DR in Karnataka, as shown in Figure 6, and it thus has more availability than other end uses. Finally, agricultural loads tolerate a longer period between the DR event and its associated recovery; the recovery time for agriculture can be up to 24 hours instead of just 2-4 hours for the other end uses. There are significant operational implications associated with end uses differing operational constraints. 
In the High Solar scenario, DR increases agricultural load in midday when solar generation is high and net load is low. However, commercial space cooling load is unable to shift its consumption schedule over such a long period because of its four-hour recovery constraint, and its lack of recovery available during the hottest time of the day; rather, commercial space cooling can shift to mitigate ramping requirements in either of the shoulder periods (morning or evening) but cannot reduce load en masse during midday. The sum of all DR end uses increases load during the high solar resource portions of the day and decreases load during the evening, as shown in Figure 9.

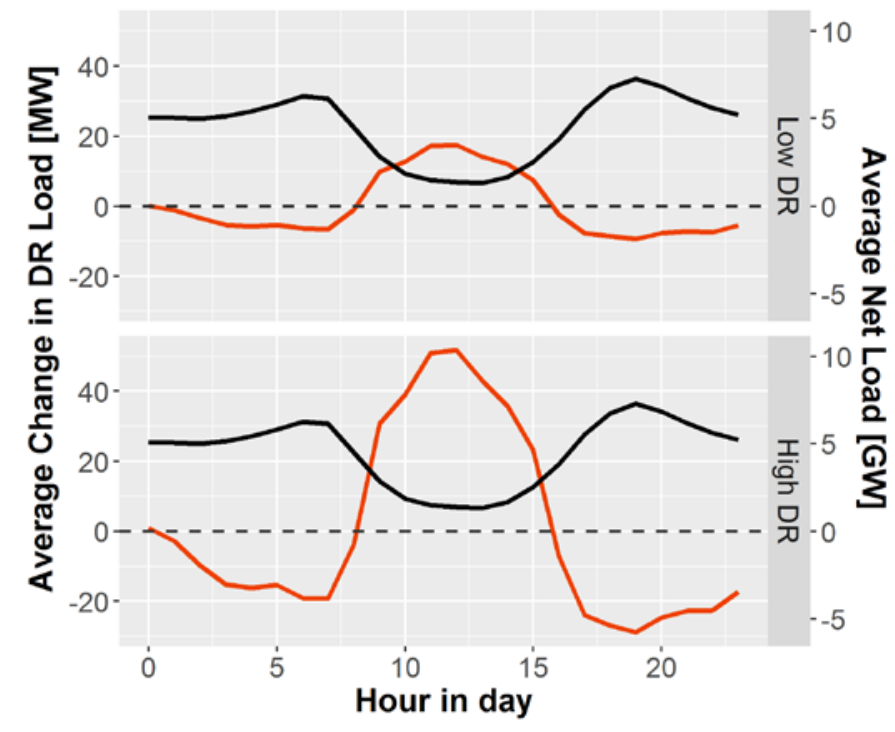

- Avg. Change in Load, all DR - Avg. Net Load

Figure 9. Daily DR operations averaged for each hour of the day over all nodes and all end uses in the High DR and Low DR, HighSR scenarios, as compared to net load

Note - The scale of the net load is different from the DR to help illustrate the signal to which the DR is reactinga need for increased generation during the middle of the day and reduction in the morning and evening. 
The seasonal variation in DR usage differs across end uses. Industrial motor DR is relatively consistent throughout the course of the year. On the other hand, almost twice as much industrial $\mathrm{AC}$ demand response is dispatched between July and September as compared to February, as shown in Figure 11.
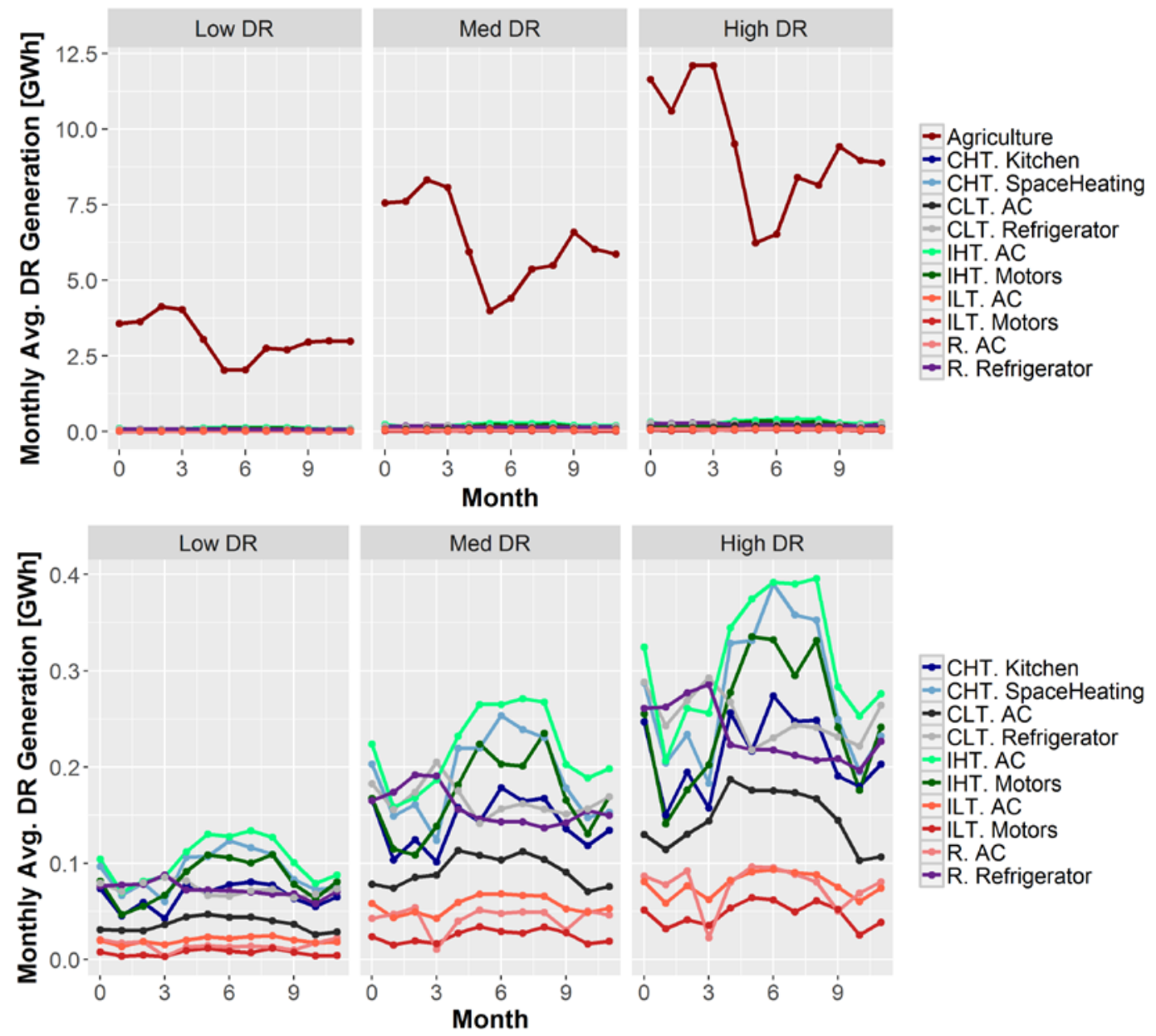

Figure 10. Monthly DR utilization per end use for all end uses (top) and excluding agriculture (bottom)

\subsection{System Impacts of Introducing Demand Response}

The addition of DR increases system flexibility, which provides several system benefits. First, DR facilitates a fuel shift from high marginal cost subcritical coal and diesel, the two most expensive marginal cost generation types on Karnataka's electricity system, to lower or zeromarginal-cost generation types, including wind, solar PV, and supercritical coal. Displacing thermal generation with wind and solar inherently reduces curtailment of these resources, leading to overall higher utilization of these resources. With one notable exception, the overall fuel shifting trends are comparable in the Low DR, Medium DR and High DR scenarios, as shown in Figure 12; while the Low DR and Medium DR scenarios exclusively replace fossil generation with renewable generation, the High DR scenario replaces some subcritical coal and diesel generation for less-expensive supercritical coal generation. 

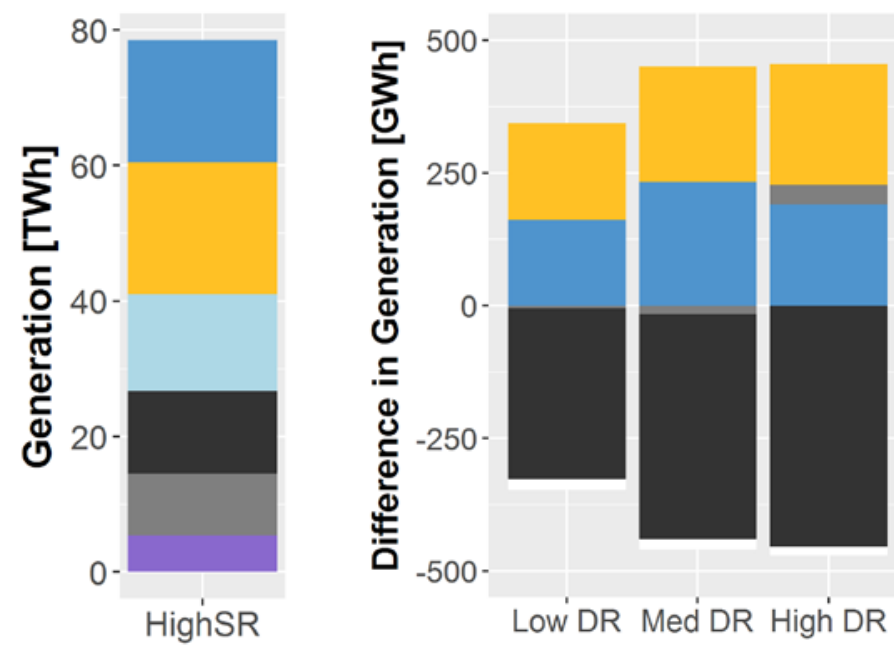

Wind

Solar-PV

Gas CT

Gas CC

Hydro

Sub-Coal

Super-Coal

Nuclear

Other

Figure 11. Karnataka's baseline generation profile (left) compared to the change in generation due to the introduction of DR (right) in the High Solar scenario

The shift from fossil fuel generation to RE generation has several positive system impacts. First, VRE curtailment is reduced by between 343 gigawatt-hours (GWh) in the Low DR scenario and $418 \mathrm{GWh}$ in the High DR scenario, from a total of 3,212 GWh without DR. Further, the decrease in thermal generation reduces fuel requirements and their associated fuel costs by $0.15-0.18$ crore $^{1}\left(22,000-26,000 \mathrm{USD}^{2}\right)$, as well as startup and shutdown costs by up to 6,000 INR (87 USD), from a total cost of 7.39 crore without DR, Table 3 . These savings represent $2 \%-3 \%$ of total production costs. However, perhaps more importantly, the Low DR scenario provides the highest value to the system per unit of DR generation, as shown in Figure 13. While additional DR availability reduces absolute production costs, the incremental cost reduction decreases.

Table 3. Total cost reduction from each DR scenario

\begin{tabular}{|c|c|c|c|}
\hline DR Scenario & $\begin{array}{c}\text { Generation Cost } \\
\text { Reduction (crore) }\end{array}$ & $\begin{array}{c}\text { Start and Shtudown Cost } \\
\text { Reduction (crore) }\end{array}$ & $\begin{array}{c}\text { Total Cost Reduction } \\
\text { (crore) }\end{array}$ \\
\hline Low DR & 0.15 & 0.006 & 0.15 \\
Med DR & 0.18 & 0.005 & 0.18 \\
High DR & 0.16 & 0.002 & 0.16 \\
\hline
\end{tabular}




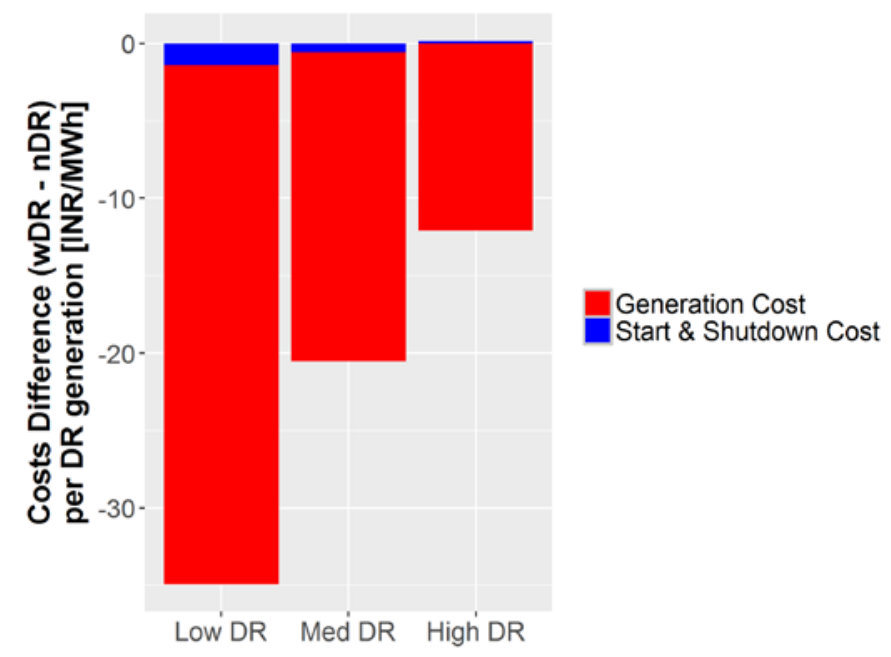

Figure 12. Marginal reduction in production costs per unit of DR activity for increasing DR availability

Changes in production costs are shown as a fraction of DR activity for each DR increment.

1 One crore Indian rupee $=10$ million Indian rupees

2 One Indian rupee $=0.015$ USD

$\mathrm{wDR}=$ with $\mathrm{DR}$ and $\mathrm{nDR}=$ no $\mathrm{DR}$

The DR-enabled fuel shift from thermal to renewable generation also reduces carbon dioxide $\left(\mathrm{CO}_{2}\right)$ emissions by $0.5-0.6$ million tons, in a system that emits approximately 23 million tons of $\mathrm{CO}_{2}$. In Karnataka, high marginal cost resources are also emissions-intensive resources; therefore, on this specific system, shifting to lower cost resources tends to reduce emissions. However, other systems (e.g., those with high gas generation) may not find similar emissions reductions (Stoll, Buechler, and Hale 2017). These emissions reductions represent a 2\%-3\% reduction in emissions in Karnataka. Although the absolute emissions reductions increase with increased DR availability, the relative emissions reductions, calculated as a fraction of DR generation, decreases with increasing DR availability, as shown in Figure 14. Thus, like production costs, the deployment in the Low DR scenario provides the largest relative emissions reductions on this system. Incremental additions of DR reduce absolute $\mathrm{CO}_{2}$ emissions further, but to a lesser extent per MWh of DR generation. 


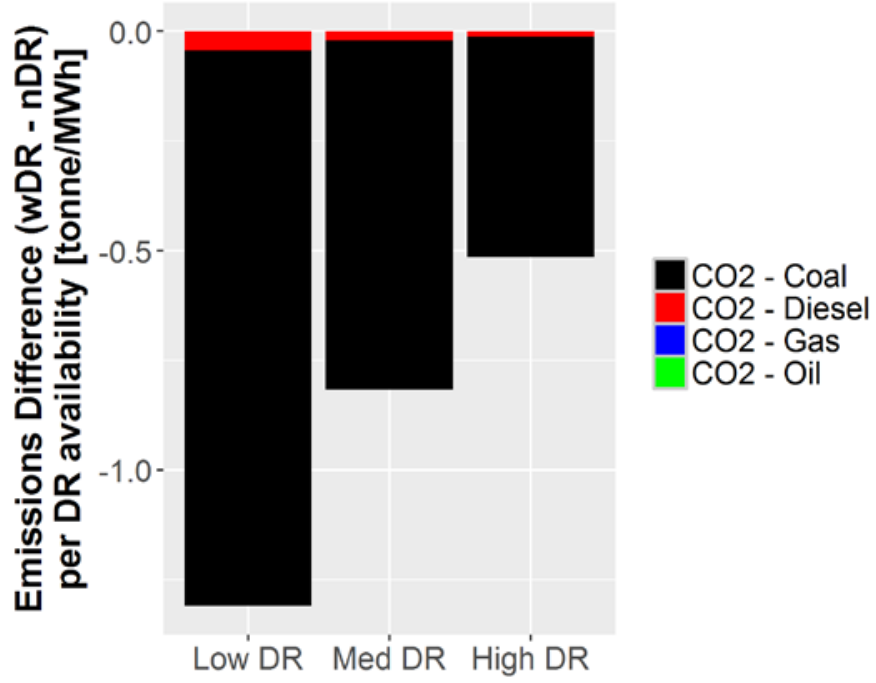

Figure 13. Marginal reduction in $\mathrm{CO}_{2}$ emissions that are due to the introduction of $\mathrm{DR}$, per unit of DR activity

Changes in emissions are shown as a fraction of DR activity for each DR increment.

\subsection{System Operational Implications of Integrating Demand Response}

In addition to enabling a fuel shift from high-marginal-cost and emission-intensive generation types to zero-marginal-cost emissions-free generation, DR changes how individual generation assets are operated. Demand response has the most profound effect on diesel assets, which are the most expensive generation assets to operate, and they typically fill a similar role as DR: quick starting generation utilized for a short amount of time. These units are typically only used a few times per year for only a few hours at a time. Demand response reduces diesel's plant load factor, the number of plant starts, and the percentage of time spent at minimum stable level, as shown in Figure 15, by displacing the need for these units entirely during 50 or more hours per year.
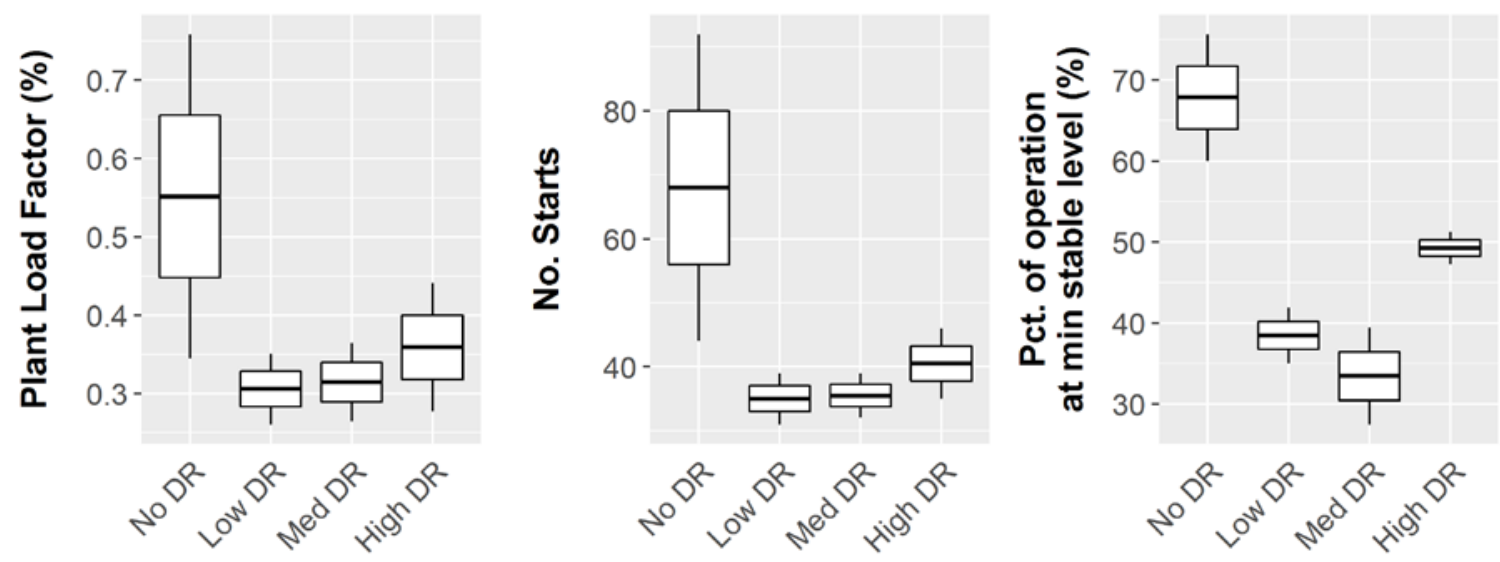

Figure 14. Impact of DR on diesel operations: Plant load factor (left), number of starts (center), and percentage of time at minimum stable level (right) 
The impacts of DR integration on subcritical coal and supercritical coal asset operations are in most cases negligible. There is no significant impact on the plant load factor of these resources and only moderate impacts on the time spent at minimum stable level and a slight increase in the number of starts of subcritical coal. Given the amount of DR generation relative to that of coal, it is not possible to draw significant conclusions about the impacts of DR on coal as there is not a strong enough effect. The one exception is a reduction in time spent at minimum load for supercritical coal, as shown in Figure 16. Demand response was able to reduce the amount of time these resources spent at minimum stable level, thereby improving the overall efficiency of these plants.
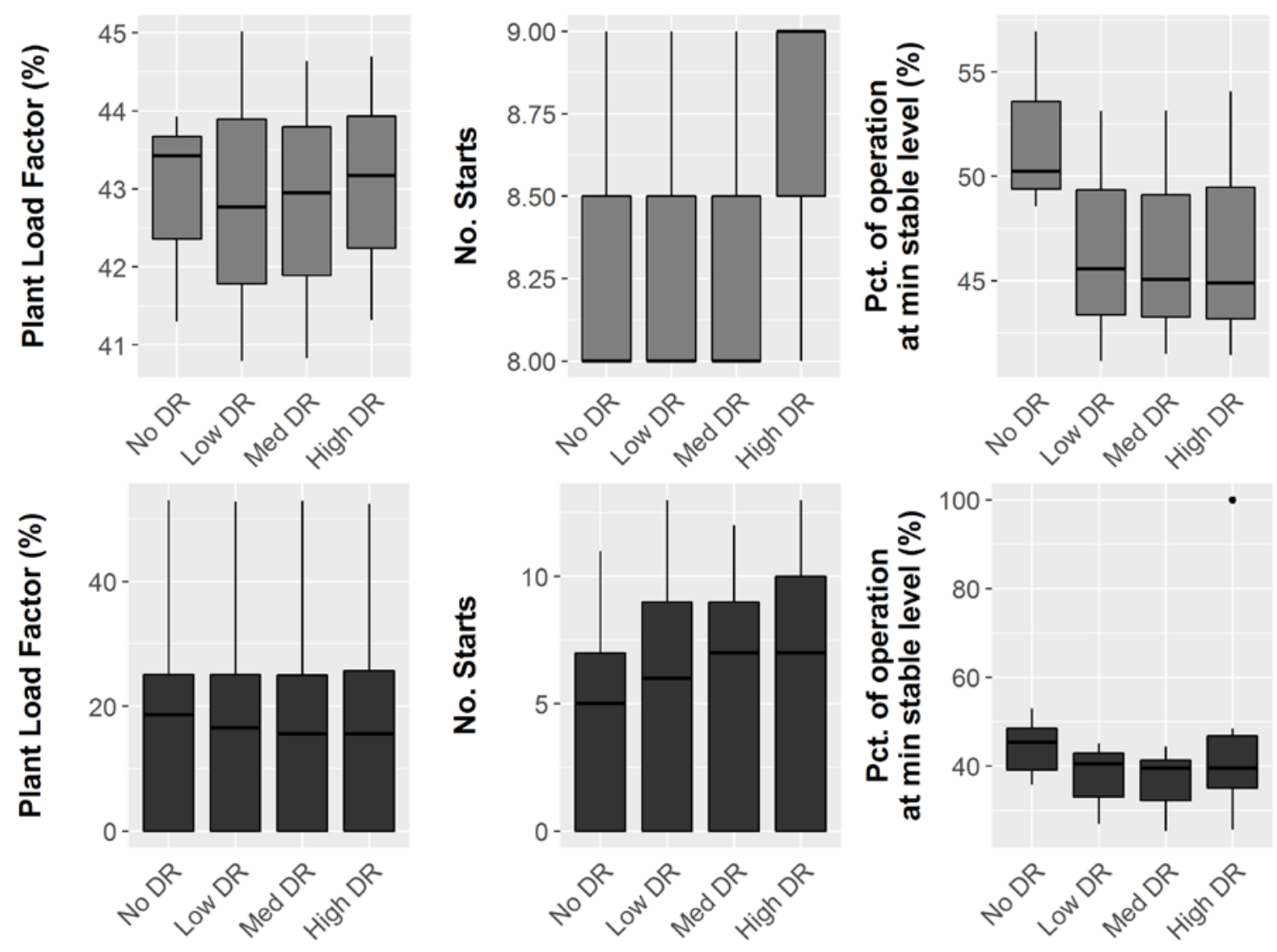

Figure 15. Impact of DR on supercritical coal (top) and subcritical coal (bottom) operations for plant load factor (left), number of starts (center), and percentage of time at minimum stable level (right)

The reduction in time spent at minimum stable level is perhaps the most important operational impact from a VRE integration perspective. An asset operating at its minimum stable level cannot ramp down without having to shut down, and thus is more likely to require VRE curtailment during those times. A reduction in time spent at minimum stable level gives the system operator the latitude to ramp up or down between the minimum and maximum stable levels, resulting in greater overall flexibility (as long as the asset is not being dispatched at its maximum stable level).

Thus, as with diesel, the system operator has more latitude while dispatching coal assets, by being able to ramp them up or down. Interestingly, the number of plant starts for subcritical coal 
and supercritical coal facilities increases because of DR integration. However, the cost burden associated with increased coal starts are outweighed by the cost reductions associated with the reduced diesel starts.

\subsection{Economic Value of Demand Response}

System planners must balance their system capacity requirements, given the system's peak load and fixed reserve margin, with capacity provision across the generation fleet. An asset's capacity credit represents the fraction of its installed capacity that should be counted toward meeting the system capacity requirement. Only a fraction of DR capacity can be counted toward system capacity requirements, as it is not consistently available as a result of time-varying load profiles and consumer-tolerance constraints. To calculate the capacity credit of DR, we sum the DR availability during the top 100 load hours, which then represents the fraction of DR capacity that could displace conventional capacity and contribute to the system capacity requirement. This method has been shown to approximate more rigorous methods for variable generation (Madaeni, Sioshansi, and Denholm 2012). The capacity credit for DR end uses range from 10\% to $30 \%$, as shown in Figure 17 . We account for all energy limitations outlined in Table 2 when calculating the capacity credit, but we do not account for recovery in this calculation. This assumes this calculation approximates the loss of load probability and assumes these resources would be called only in the event of a grid shortage. Such events typically last only a short time and are called only infrequently, so the DR constraints listed in Section 2.6 would not be binding.

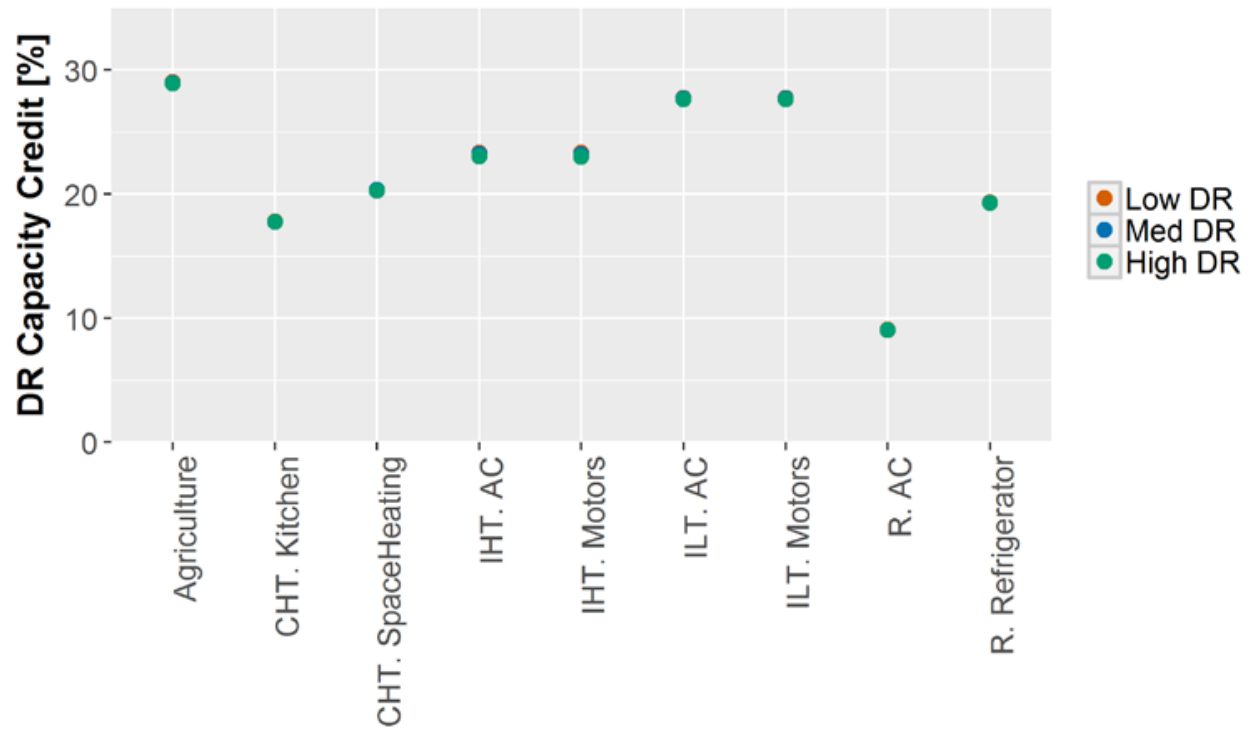

Figure 16. Capacity credit of each DR end use

The capacity value monetizes an asset's capacity provision to represent the avoided cost of building new capacity resources. Capacity value is the product of capacity credit and the lowest capital cost generation type (a gas combustion turbine in this case), and thus mirrors the capacity credit values. The resulting capacity values for DR range from approximately $500 \mathrm{INR} / \mathrm{MWh}$ to $1,500 \mathrm{INR} / \mathrm{MW}$. 
Agriculture has the highest capacity credit and value among end uses; agricultural DR availability is coincident with high-load periods of the year and is more available than other end uses. However, the difference between agricultural capacity credit and sectoral capacity credit is significantly smaller than the difference between agricultural utilization rates and other sectoral utilization rates. While agriculture's contribution to reduced production costs and emissions outweighs that of other end uses substantially, the value it provides to the system from a capacity perspective is only marginally greater than that of other end uses.

Increasing DR penetrations have a negligible impact on end-uses' capacity credit because of the overall small size of the resource; even the High DR scenario does not saturate the load reduction potential in the top 100 hours. Capacity credit could fall with increasing DR penetrations if there were sufficient DR to fully reduce the load in some of the top 100 hours. However, the consistency in capacity credit observed in Figure 17 suggests DR's contribution to capacity adequacy has not yet exceeded the system's capacity requirements during the specified high-load periods.

The cumulative value that DR provides to the electricity system is the sum of its (1) contribution to capacity requirements, (2) the reduction in production costs and emissions it induces by enabling a shift from high-marginal-cost fossil generation to zero-marginal-cost renewable generation, and the (3) reduction in electricity prices it induces by shifting load from high price periods to low price periods. This system wide-value can then be compared to the revenue a DR aggregator may expect to earn by selling DR services in the electricity market. Demand response revenues from price arbitrage are calculated by subtracting the consumption costs during recovery in low-cost hours from the avoided load charges in high-cost hours. As shown in Figure 19, net revenues per MWh of DR generation range from $440 \mathrm{INR} / \mathrm{MWh}$ to 900 INR/MWh for commercial end uses, from 520 to 970 IRN/MWh for industrial end uses, 390-790 INR/MWh for residential end uses, and from 370 to $430 \mathrm{INR} / \mathrm{MWh}$ for agricultural end uses. Interestingly, these values do not change significantly at increasing DR penetrations, again indicating these DR scenarios are not saturating the need for flexibility in the Karnataka power system at high VRE penetrations. Summing across end uses and total generation from DR, a DR program could expect to earn net revenues that range from 2.1 crore in the Low DR scenario to 5.7 crore in the High DR scenario. 


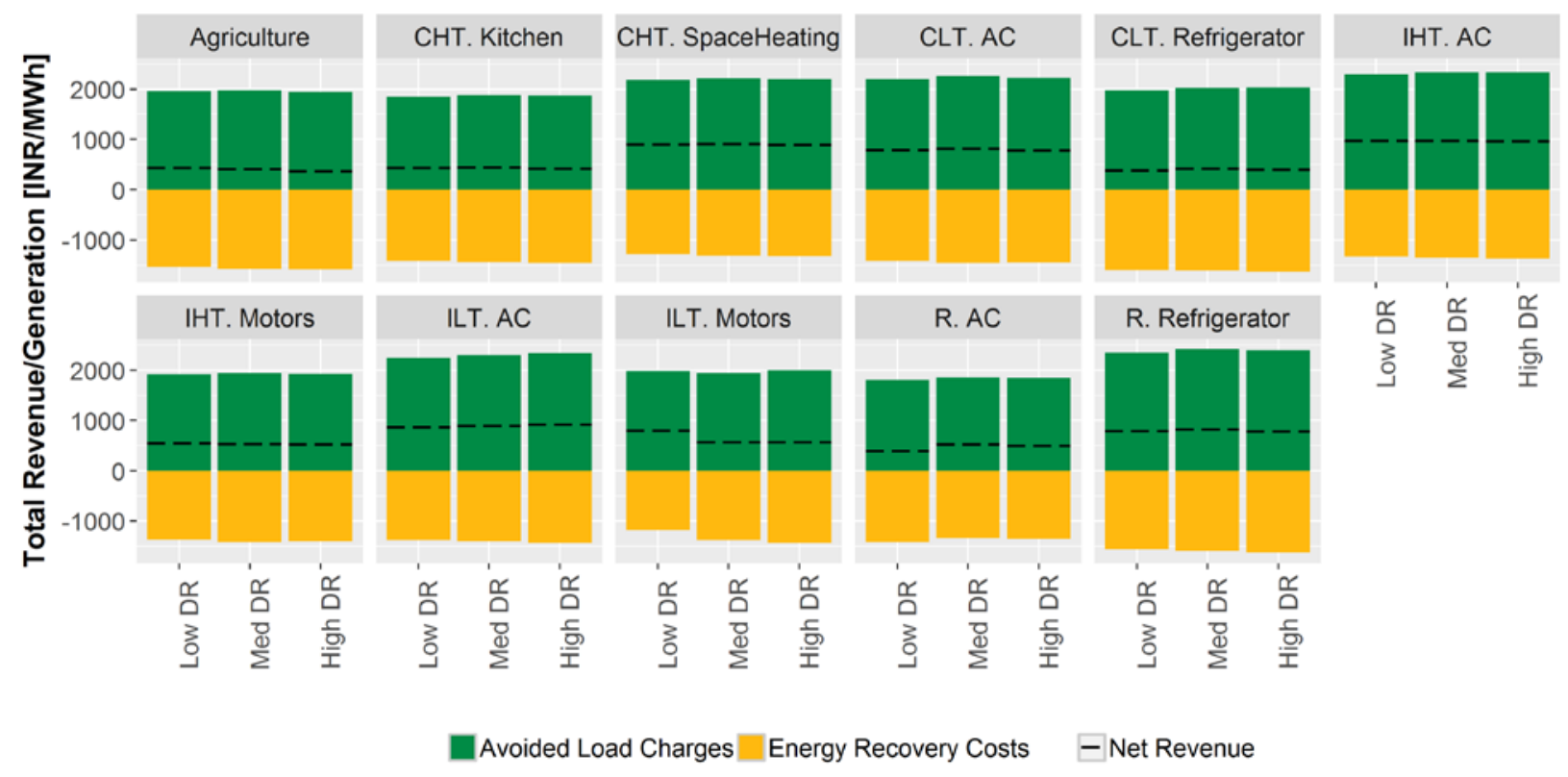

Figure 17. Demand response revenues per MWh of DR generation, by end use for each DR scenario

\subsection{Impact of Demand Response in Different Renewable Energy Scenarios}

The previous sections focused on the impact of DR in the High Solar scenario, which represents India's targeted 2022 buildout. However, DR has different impacts in systems with alternative RE penetrations - demonstrating how DR can be used to help integrate wind and solar differently - and how these impacts change at increasing VG penetration. Karnataka's generation by technology type is shown in Figure 20 for each renewable penetration scenario. ${ }^{4}$ Generation in the No New RE scenario is dominated by thermal generation, with only modest contributions from wind and solar PV (13\% VRE penetration before DR). The contribution from wind is most pronounced in the High Wind scenario (53\% VRE penetration before DR), while the contribution from solar PV is most pronounced in the High Solar (48\% VRE penetration before DR) and High RE (61\% VRE penetration before DR) scenarios. Nuclear and hydro generation remains constant across the RE scenarios.

\footnotetext{
${ }^{4}$ Differences in cumulative generation across the renewable energy scenarios are a result of imports and exports that differ between Karnataka and its neighbors, as load is served in all scenarios.
} 


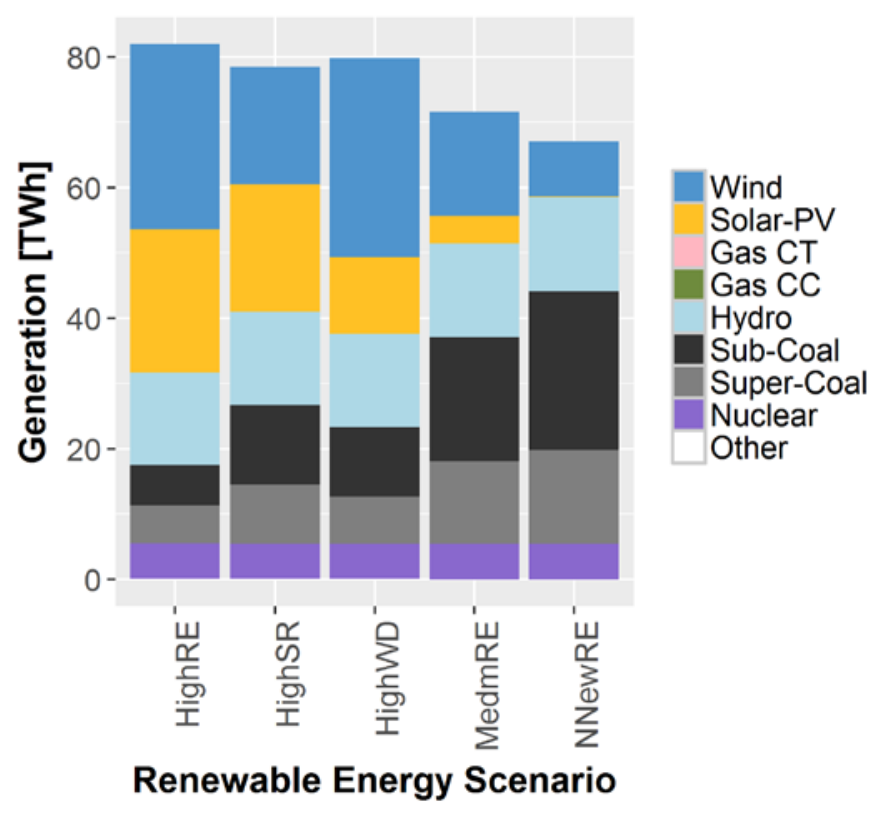

Figure 18. Generation in each RE scenario in Karnataka, before DR is added to the system

Demand response is most impactful in scenarios with significant VRE penetrations, but it does have a slight, though negligible, impact in the No New RE scenario, as shown in Figure 21. The key impact of DR is consistent across RE scenarios: high-cost subcritical coal and diesel generation is replaced primarily with zero-marginal-cost renewable generation, as well as lowercost supercritical coal generation in the Medium RE and No New RE scenarios.

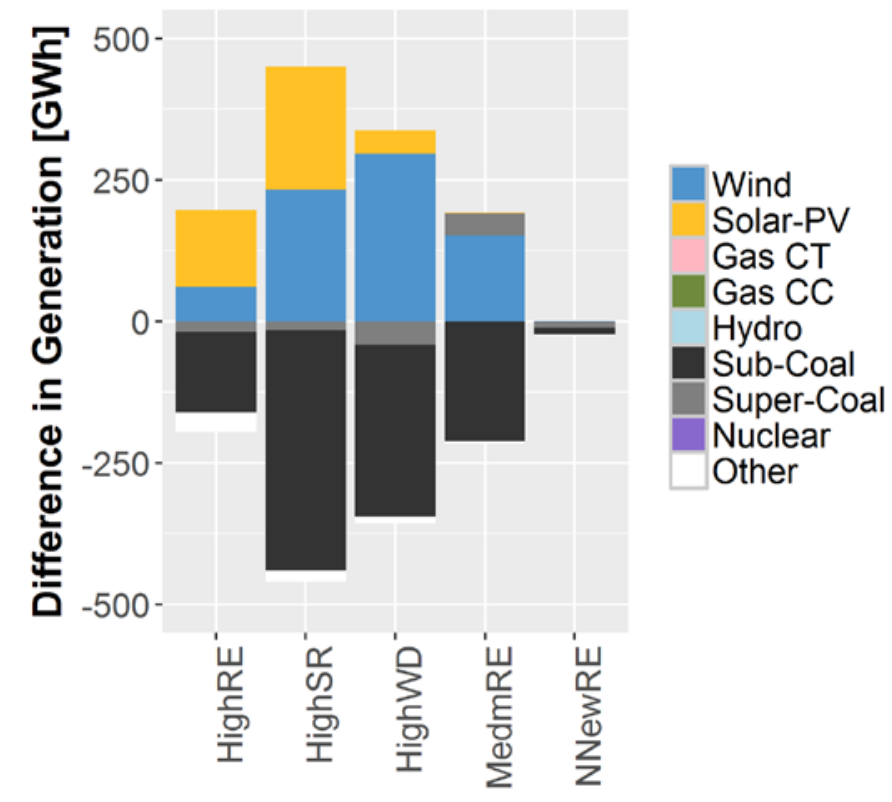

Figure 19. Change in generation after introducing the medium DR availability scenario for each RE penetration scenario

The implications of demand-response-enabled fuel shifting are consistent with the previous discussion; displacing high-marginal-cost and emissions-intensive subcritical coal with zero- 
marginal-cost and emissions-free renewable generation reduces production costs and greenhouse gas emissions proportionally to the change in generation shown in Figure 21. The main difference across renewable scenarios is the variable need for integrating renewables. While VRE is present in the NNewRE scenario, all VRE is integrated before DR is added, so DR is used to obtain marginal cost reductions by switching load between higher- and lower-cost coal. In the High Solar scenario, which had much higher amounts of curtailment, DR was able to help integrate VRE resources by enabling greater flexibility on the system.

The utilization rates across end uses are generally consistent among the RE penetration scenarios, as shown in Figure 22. Most significantly, agricultural load shifting dominates over other end uses in all RE scenarios, primarily because of its more relaxed utilization constraints and its higher availability, as discussed in Section 3.1. There is a slight increase in DR utilization in higher penetration scenarios, particularly those dominated by solar power.

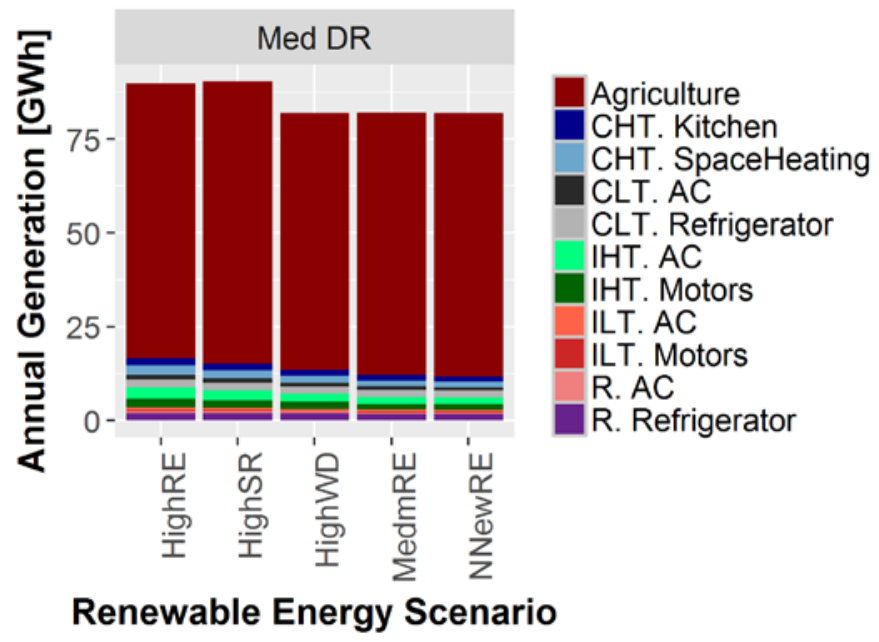

Figure 20. Annual activity of each DR end use in the Medium DR scenario, for each RE penetration scenario

Interestingly, while annual DR activity is similar across RE scenarios (Figure 22), the fuelshifting impacts of DR differ across RE scenarios (Figure 21). As the RE penetration increases, the opportunity for DR to enable fuel shifting from high-marginal cost thermal generation to zero-marginal-cost renewable generation also increases as a result of typically increasing curtailment. However, even in low renewable penetration scenarios, DR is dispatched because it can provide other services to the system.

More specifically, in high-solar scenarios, DR is dispatched to increase mid-day load, when solar generation is high, and to smooth ramping needs during the shoulder periods, thereby reducing VRE curtailment. However, DR dispatch in the Medium RE and No New RE scenarios, while comparable in overall magnitude (Figure 22) to the high-renewable scenarios, has much less correlation with the net load profile, as shown in Figure 23, and no clear yearly pattern as is seen in high solar scenarios. The DR resources are still providing value to the grid in these cases, but the value is based more on the slight benefits of fuel shifting and local grid issues, rather than more broadly to integrate renewable generation. 


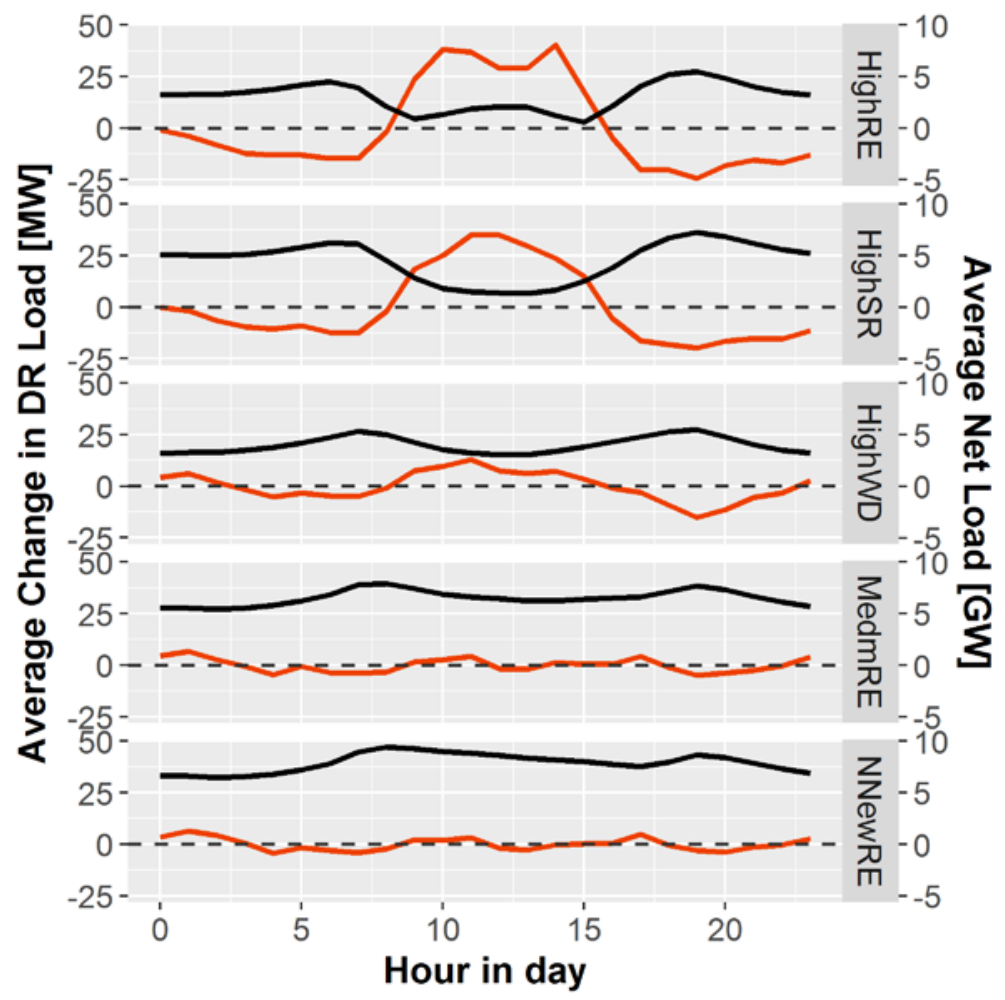

-Avg. Change in Load, all DR-Avg. Net Load

Figure 21. Daily DR operations averaged overall all nodes and end uses as compared to the net load in the Medium DR scenario, for each RE penetration scenario 


\section{Conclusions}

The results of exploring DR integration in BESCOM's territory within Karnataka provide several conclusions that are relevant for power system stakeholders, as well as policymakers and regulators who are designing DR programs.

\section{Understanding the applicable operational constraints representing consumer preferences has a material impact on DR operations and its subsequent value to the grid.}

The constraints imposed on each DR end use, particularly intraday constraints, had a large impact on the ability of end uses to integrate VRE. Accurately accounting for consumer preferences and tolerances for load shifting led to low overall utilization of DR potential, but in a more realistic manner. End uses, such as agriculture, without intraday constraints and with longer shifting periods had significantly higher utilization rates than other end uses, largely because of more relaxed operational constraints. The strict intraday constraints imposed on most end uses are the most impactful consideration for determining DR utilization and value, more so than the DR availability. As a result, when attempting to understanding the value DR could provide to electricity system operations, BESCOM and other policymakers should account for consumer requirements in a realistic manner and include those as DR constraints. While consumers may have some flexibility if appropriately incentivized that could be explored, accounting for more accurate constraints on consumer behavior is important. Additional work is required to develop accurate consumer tolerance parameters and constraints for DR.

\section{Demand response reduces production costs and emissions given BESCOM's specific electricity grid characteristics.}

Adding DR enables fuel switching from high-marginal-cost and emissions-intensive thermal generation to zero-marginal-cost emissions-free renewable generation. This fuel switch has numerous positive follow-on impacts, including reduced production costs and emissions. Adding flexibility to the demand side is an important and impactful tool for making better use of supplyside assets.

\section{Demand response provides the largest benefits to systems with high renewable energy (RE) penetrations and inflexible generation mixes, exemplified by the high renewable future of BESCOM's territory.}

While DR is utilized to a similar degree across RE scenarios, the impact of DR utilization on system metrics such as production cost and emissions impacts increases at high RE penetrations. Further, in the BESCOM territory, which is relatively inflexible (i.e., limited ramping capability), DR is particularly helpful for integrating solar PV which introduces daily variation in net load. Demand response will become an increasingly important tool as penetrations of renewables increase, and in systems which rely heavily on peaking units. Planners and operators in jurisdictions facing high RE penetrations in the coming decades should consider DR as an integration option. 


\section{Demand response increases a system's operational flexibility, which in turn reduces}

the need for expensive but flexible diesel dispatch.

The addition of DR to the electricity system reduces the use of diesel generators, as DR provides a very similar use to systems operators that is due to the fast response rate and short time scales on which these resources operate. Additionally, on the BESCOM system, coal generators spent less time at minimum load, which is an inflexible operational state. By reducing time spent at minimum load, the system operator has access to greater asset flexibly and can reduce out-ofmerit commitments. System operators contending with particularly inflexible electricity systems could leverage DR to access greater system flexibility at a lower cost than utilizing peaker facilities.

\section{The first increment of $D R$ provides the highest relative value to the system.}

Though increasing DR availability reduces production costs and greenhouse gas emission in absolute terms, each incremental DR addition has a smaller marginal impact than the first DR increments. BESCOM, or other utilities considering DR programs, would see system benefits, even if the initial subscription were low or if only some customers could be enrolled at the start of a program. As such, introducing initial programs are recommended to begin seeing the impacts of DR programs. Demand response programs will provide a net benefit to the system so long as the cost to operate the program is below the marginal net cost reduction per MWh of demand response provided. 


\section{References}

Energy Exemplar. 2018. "Energy Market Modelling.” 2018. http://energyexemplar.com/.

Karali, N, N Abhyankar, and A Khandekar. 2019. "Bottom-up Estimation of the End-Use Level Load and Demand Response Potential in India." Working Paper 008. Lawrence Berkeley National Laboratory.

Madaeni, S. H., R. Sioshansi, and P. Denholm. 2012. "Comparison of Capacity Value Methods for Photovoltaics in the Western United States.” https://doi.org/10.2172/1046871.

O'Connell, Niamh, Elaine Hale, Ian Doebber, and Jennie Jorgenson. 2015. "On the Inclusion of Energy- Shifting Demand Response in Production Cost Models : Methodology and a Case Study." NREL National Renewable Energy Laboratory, Office of Energy Efficiency \& Renewable Energy, no. July: 1-42.

O’Connell, Niamh, Pierre Pinson, Henrik Madsen, and Mark O’Malley. 2014. "Benefits and Challenges of Electrical Demand Response: A Critical Review." Renewable and Sustainable Energy Reviews 39: 686-99. https://doi.org/10.1016/j.rser.2014.07.098.

Palchak, David, Jaquelin Cochran, Ali Ehlen, Brendan McBennett, Michael Milligan, Ilya Chernyakhovskiy, Ranjit Deshmukh, et al. 2017a. "Greening the Grid: Pathways to Integrate 175 Gigawatts of Renewable Energy into India’s Electric Grid, Vol. INational Study." Vol. I.

- 2017b. "Greening the Grid: Pathways to Integrate 175 Gigawatts of Renewable Energy into India's Electricity Grid, Vol. II - Regional Study.” Vol. II.

Palchak, David, Jaquelin Cochran, Analiese Ehlen, Brendan McBennett, Michael Milligan, Chernyakhovskiy, Ilya, et al. 2017. "Greening the Grid: Pathways to Integrate 175 Gigawatts of Renewable Energy into India’s Electric Grid, Regional Study: Karnataka.” https://doi.org/NREL/TP-6A20-70950.

Siano, Pierluigi. 2014. "Demand Response and Smart Grids-A Survey." Renewable and Sustainable Energy Reviews 30: 461-78. http://dx.doi.org/10.1016/j.rser.2013.10.022.

Stoll, Brady, Elizabeth Buechler, and Elaine Hale. 2017. "The Value of Demand Response in Florida." Electricity Journal 30 (9): 57-64. https://doi.org/10.1016/j.tej.2017.10.004. 